\title{
A MODIFIED SCHUR ALGORITHM AND AN EXTENDED HAMBURGER MOMENT PROBLEM
}

\author{
OLAV NJASTAD
}

\begin{abstract}
An algorithm for a Pick-Nevanlinna problem where the interpolation points coalesce into a finite set of points on the real line is introduced, its connection with certain multipoint Padé approximation problems is discussed, and the results are used to obtain the solutions of an extended Hamburger moment problem.
\end{abstract}

\section{INTRODUCTION}

The Pick-Nevanlinna problem is the following: Let $\left\{z_{\alpha}: \alpha \in A\right\}$ be a set of distinct points in the open upper half-plane $H_{+}^{0}=\{z: \operatorname{Im} z>0\}$, and let $\left\{w_{\alpha}: \alpha \in A\right\}$ be a set of points in $\mathbf{C}$. Find a function $F(z)$ which is analytic in $H_{+}^{0}$ such that $\operatorname{Im} F(z) \geq 0$ for $z \in H_{+}^{0}$ and $F(z)=w_{\alpha}$ for all $\alpha \in A$. (A function $F(z)$ which is analytic for $z \in H_{+}^{0}$ with $\operatorname{Im} F(z) \geq 0$ is called a Nevanlinna function.) The problem was solved by Pick [27, 28] in the case that $A$ is finite, by Nevanlinna $[15,16]$ in the case that $A$ is countable, and by Krein and Rekhtman [14] in the general case.

A variant of the problem for finite or countable $A$ arises when all points $z_{\alpha}$ coalesce to a single point $a$, and given values $w_{\alpha}$ at the points are replaced by the Taylor coefficients at $a$. A problem closely related to this is Carathéodory's coefficient problem (see $[2,3,33])$ : Given a finite sequence $\left\{\gamma_{0}, \ldots, \gamma_{m}\right\}$ or an infinite sequence $\left\{\gamma_{n}: n \in \mathbf{N}\right\}$, find a function $F(z)$ which is analytic in the open unit disc $D^{0}=\{z:|z|<1\}$ such that $\operatorname{Re} F(z) \geq 0$ for $z \in D^{0}$ and $F(z)=\sum_{k=0}^{m} \gamma_{k} z^{k}+\sum_{k=m+1}^{\infty} \delta_{k}^{(m)} z^{k}$, or $F(z)=\sum_{k=0}^{\infty} \gamma_{k} z^{k}$. (For historical remarks on this problem, see [12].)

By introducing the linear fractional transformation $F \rightarrow \frac{1-F}{1+F}$ we can reformulate the requirement $\operatorname{Re} F(z) \geq 0$ to read $|F(z)| \leq 1$. (A function $F(z)$ which is analytic for $z \in D^{0}$ is called a Carathéodory function if $\operatorname{Re} F(z) \geq 0$ for $z \in D^{0}$, or a Schur function if $|F(z)| \leq 1$ for $z \in D^{0}$.) Schur [31] invented an algorithm called the Schur algorithm to deal with this problem. The technique was adapted by Nevanlinna to deal with the Pick-Nevanlinna problem.

Received by the editors August 21, 1989.

1980 Mathematics Subject Classification (1985 Revision). Primary 30E05, 30E15, 41A21.

Key words and phrases. Asymptotic expansions, multipoint Padé approximants, extended Hamburger moment problem. 
For modern applications of the Schur algorithm and variants, see the collection [5]. For an operator theoretic approach, see, e.g., [29, 30].

A modification of the Pick-Nevanlinna problem with coinciding points $z_{n}=$ $a, n \in \mathbf{N}$, arises when the point $a$ is moved to the boundary of the region of definition of $F(z)$, i.e., to the real axis. The Taylor series expansion then has to be replaced by the asymptotic series expansions $\sum_{k=0}^{\infty} \gamma_{k}(z-a)^{k}$ at $a$, valid in arbitrary angular regions $R_{a, \delta}=\{z: \delta<\operatorname{Arg}(z-a)<\pi-\delta\}, \delta>0$. If moreover the point $a$ is moved to the point at infinity, then we obtain the following modified Pick-Nevanlinna problem: Given a sequence of numbers $\left\{\gamma_{n}: n \in \mathbf{N}\right\}$, find a Nevanlinna function $F(z)$ with the asymptotic expansion $F(z) \approx \sum_{k=0}^{\infty} \gamma_{k} z^{-k}$ at $\infty$, valid in every angular region $R_{\delta}=\{z: \delta<\operatorname{Arg} z<$ $\pi-\delta\}, \delta>0$. This problem is equivalent to the classical Hamburger moment problem (see, e.g., $[1,6,32])$.

If the points $z_{n}, n \in \mathbf{N}$, coalesce into the two points 0 and $\infty$ according to the rule $z_{2 m}=0, m=1,2, \ldots, z_{2 m+1}=\infty, m=0,1,2, \ldots$, then the corresponding modification of the Pick-Nevanlinna problem can be formulated as follows: Let $\left\{\gamma_{n}: n=0, \pm 1, \pm 2, \ldots\right\}$ be a bi-infinite sequence of numbers. Find a Nevanlinna function $F(z)$ with the asymptotic expansions $F(z) \approx$ $\sum_{k=0}^{\infty} \gamma_{k} z^{-k}$ at $\infty, F(z) \approx \sum_{k=1}^{\infty} \gamma_{-k} z^{k}$ at 0 , in every angular region $R_{\delta}=$ $\{z: \delta<\operatorname{Arg} z<\pi-\delta\}$. This problem is equivalent to the strong Hamburger moment problem. For definition and general treatment of this problem, see, e.g., $[10,11,25]$. The connection between the strong moment problem and the asymptotic expansions problem was treated in [10] for the case that the moment problem is nonsingular, and in [20-23] for the general case.

Again consider the sequence $\left\{z_{n}: n \in \mathbf{N}\right\}$, and now let $z_{n}, n=1,2, \ldots$, coalesce into $p$ points $a_{1}, \ldots, a_{p}$ on the real axis as follows: $z_{p q+1}=a_{1}, z_{p q+2}$ $=a_{2}, \ldots, z_{p q+p}=a_{p}, q=0,1,2, \ldots$ A corresponding Pick-Nevanlinna problem can be formulated as follows: Let $\left\{\gamma_{j}^{(i)}: j=0,1,2, \ldots\right\}, i=$ $1, \ldots, p$, be $p$ sequences of given numbers. Find a Nevanlinna function $F(z)$ which has the asymptotic expansions $F(z) \approx \sum_{j=0}^{\infty} \gamma_{j}^{(i)}\left(z-a_{i}\right)^{j}$ at $a_{i}$ in the angular regions $R_{i, \delta}=\left\{z: \delta<\operatorname{Arg}\left(z-a_{i}\right)<\pi-\delta\right\}, \delta>0, i=1, \ldots, p$. This problem will later be shown to be related to the extended Hamburger moment problem.

The use of the Schur algorithm and its variants has two steps. The first step is to successively construct rational functions $f_{n}(z)$ solving the truncated (finite) interpolation problem. (In the classical Hamburger moment case, e.g., this consists of finding for each $n$ a rational function with power series expansion

$$
f_{n}(z)=\sum_{k=0}^{2 n} \gamma_{k} z^{-k}+\sum_{k=2 n+1}^{\infty} \delta_{k}^{(n)} z^{-k}
$$

This is the Pade approximation problem for the series $\sum_{k=0}^{\infty} \gamma_{k} z^{-k}$, or for the solutions of the original problem, if they exist.) The second step is to obtain from the sequence $\left\{f_{n}(z)\right\}$ one or more of the original functions $F(z)$ which solve the nontruncated problem, if they exist. 
In this paper we treat the above-mentioned version of the Pick-Nevanlinna problem related to the extended Hamburger moment problem. We describe an algorithm which generalizes the above-mentioned adaptions of the Schur algorithm. The algorithm produces certain (weak) multipoint Padé approximants $f_{n}(z)=A_{n}(z) / B_{n}(z)$ to the given power series. For reasons that will become clear later, we include a condition $F(z)=\gamma_{-1}^{(\infty)} z^{-1}+o\left(z^{-1}\right)$ at $\infty$, $z \in R_{\delta}=\{z: \delta<\operatorname{Arg} z<\pi-\delta\}, \delta>0$. (For convenience we shall also call an expansion of this form on asymptotic expansion.) We shall in this paper call this problem the modified Pick-Nevanlinna problem, and the algorithm the modified Schur algorithm (associated with the series $\gamma_{-1}^{(\infty)} z^{-1}, \sum_{j=1}^{\infty} \gamma_{j}^{(i)}\left(z-a_{i}\right)^{j}$, $i=1, \ldots, p)$.

(For treatments of multipoint Padé approximants to the series $\gamma_{-1}^{(\infty)} z^{-1}$, $\sum_{j=1}^{\infty} \gamma_{j}^{(i)}\left(z-a_{i}\right)^{j}$, see also [7, 8, 19, 24, 34].)

The rational functions $A_{n}(z) / B_{n}(z)$ are Nevanlinna functions. We show that $\left\{A_{n}(z) / B_{n}(z)\right\}$, together with a related sequence $\left\{C_{n}(z) / D_{n}(z)\right\}$, give rise to solutions of the modified Pick-Nevanlinna problem (when solutions exist).

For each $z \in H_{+}^{0}$ there exists a sequence $\left\{\Delta_{n}(z)\right\}$ of nested disks such that $f_{n}(z, \tau) \in \partial \Delta_{n}(z)$ for every $\tau \in \mathbf{R}$, where

$$
f_{n}(z, \tau)=\frac{A_{n}(z)+\tau C_{n}(z)}{B_{n}(z)+\tau D_{n}(z)} .
$$

Let $\Delta(z)$ denote the intersection $\bigcap_{n=1}^{\infty} \Delta_{n}(z)$ (which may be a closed disk or a single point). Then every Nevanlinna function $F(z)$ which satisfies $F(z)=$ $\gamma_{-1}^{(\infty)} z^{-1}+o\left(z^{-1}\right)$ and $F(z) \in \Delta(z)$ for all $z$, is a solution of the problem, and every solution has this property. Every function $f_{n}(z, \tau), \tau \in \mathbf{R}$, is a Nevanlinna function, and there exist sequences $\left\{f_{n_{k}}\left(z, \tau_{n_{k}}\right)\right\}$ converging to Nevanlinna functions $F(z)$ which satisfy $F(z)=\gamma_{-1}^{(\infty)} z^{-1}+o\left(z^{-1}\right)$ and $F(z) \in \Delta(z)$ for all $z$. Thus there exist Nevanlinna functions with the required property. These results answer in the affirmative the question of solvability of the interpolation problem (under the required conditions on the given series), and give a characterization of all the solutions. The solution is unique in the situation that $\Delta(z)$ reduces to a single point for all $z$ (the limit point case).

The extended Hamburger moment problem associated with the points $a_{1}, \ldots, a_{p}$ and sequences of real numbers $c_{0},\left\{c_{j}^{(i)}: j=1,2, \ldots\right\}, i=$ $1, \ldots, p$, is the following. Find distribution functions $\psi(t)$ (i.e.: bounded, nondecreasing functions) such that

$$
\int_{-\infty}^{\infty} d \psi(t)=c_{0}, \quad \int_{-\infty}^{\infty} \frac{d \psi(t)}{\left(t-a_{i}\right)^{j}}=c_{j}^{(i)}, \quad j=1,2, \ldots, i=1, \ldots, p .
$$

This problem was introduced and treated in $[17,18]$ in the context of the theory of positive linear functionals and of orthogonal rational functions. (In $[17,18]$ we considered the case where the distribution functions $\psi(t)$ are required to have infinitely many points of increase.) In this paper we discuss the problem 
on the basis of our results on the modified Pick-Nevanlinna problem. We point out that a sufficient condition for the existence of a solution $\psi(t)$ is that in the power series $-c_{0} z^{-1}, \sum_{j=0}^{\infty} c_{j+1}^{(i)}\left(z-a_{i}\right)^{j}$, those conditions are satisfied that lead to solutions of the modified Pick-Nevanlinna problem. The solutions are exactly those distribution functions $\psi(t)$ whose Stieltjes transform $\hat{\psi}(z)=$ $\int_{-\infty}^{\infty} d \psi(t) /(t-z)$ (which are Nevanlinna functions) satisfies $\hat{\psi}(z)=-c_{0} z^{-1}+$ $o\left(z^{-1}\right)$ and $\hat{\psi}(z) \in \Delta(z)$ for all $z$.

\section{The MOdified SchUR ALGORITHM}

Let $F(z)$ be a Nevanlinna function. We recall that this means that $F(z)$ is analytic for $\operatorname{Im} z>0$, and that $\operatorname{Im} F(z) \geq 0$ for $\operatorname{Im} z>0$. For brevity we shall write $N$-function for Nevanlinna function.

Let $a_{1}, \ldots, a_{p}$ be given distinct points on the real axis, $p \geq 3$. For convenience we assume that they are ordered by size: $a_{1}<a_{2}<\cdots<a_{p}$. We assume that $F(z)$ has the asymptotic expansions (with real coefficients)

$$
\begin{gathered}
F(z) \approx \sum_{j=0}^{\infty} \gamma_{j}^{(i)}\left(z-a_{i}\right)^{j}, \quad i=1, \ldots, p, \\
F(z)=\gamma_{-1}^{(\infty)} z^{-1}+o\left(z^{-1}\right) .
\end{gathered}
$$

(By asymptotic expansions we shall always mean expansions in the angular regions $R_{\delta}, R_{i, \delta}, i=1, \ldots, p$, defined in the introduction. For the basic definitions and results on asymptotic expansions, see, e.g., [9].)

We note that every integer $n \in \mathbf{N}$ can be written in a unique way in the form

$$
n=p \cdot q_{n}+r_{n},
$$

where $q_{n}, r_{n} \in \mathbf{N}, 1 \leq r_{n} \leq p$. When there is no danger of confusion, we write $q$ for $q_{n}$ and $r$ for $r_{n}$.

By $a_{r-1}$ we mean $a_{p}$ when $r=1$, by $a_{r+1}$ we mean $a_{1}$ when $r=p$, and so on. Statements relating to $a_{r-1}$ when $r=1 ;$ to $a_{r+1}$ when $r=p$, etc. will be understood from the context.

We shall make repeated use of the following important result.

Lemma 2.1. Let $H(z)$ be an $N$-function. Then the following statements are true:

The limit $d_{\infty}=\lim _{z \rightarrow \infty}, \operatorname{Re} z=0(-1 / z H(z))$ exists and is nonnegative, and $\operatorname{Im}\left(-1 / H(z)-d_{\infty} z\right) \geq 0$ when $\operatorname{Im} z>0$.

The limits $d_{i}=\lim _{z \rightarrow a_{i}}, \operatorname{Re}\left(z-a_{i}\right)=0\left(-\left(z-a_{i}\right) / H(z)\right)$ exist and are nonpositive for $i=1, \ldots, p$, and $\operatorname{Im}\left(-1 / H(z)-d_{i} /\left(z-a_{i}\right)\right) \geq 0$ when $\operatorname{Im} z>0$.

Proof. It follows from Julia-Carathéodory-Landau-Valiron's theorem (see, e.g., [4, p. 236]) that when $G(z)$ is an $N$-function, the limits

$$
d=\lim _{z \rightarrow \infty, \operatorname{Re} z=0} z^{-1} G(z) \text { and } d^{\prime}=\lim _{z \rightarrow 0, \operatorname{Re} z=0} z G(z)
$$


exist and are nonnegative, respectively nonpositive, and $\operatorname{Im}[G(z)-d z] \geq 0$, $\operatorname{Im}\left[G(z)-d^{\prime} z^{-1}\right] \geq 0$ when $\operatorname{Im} z \geq 0$. For any real number $a$, the function $z-a \rightarrow-1 / H(z)$ is an $N$-function. The lemma follows by application of the above-mentioned consequence of Julia-Carathéodory-Landau-Valiron's theorem to the functions $z \rightarrow-1 / H(z), z-a_{i} \rightarrow 1 / H(z)$.

We shall describe an algorithm which successively determines rational functions $f_{n}(z)$ with Taylor series expansions that progressively coincide for more and more terms with the given expansions $(2.1)$ of the $N$-function $F(z)$. We shall assume that $F(z)$ itself is not a rational function.

Since $F(z)-\gamma_{0}^{(i)}$ is an $N$-function, it follows from (2.1a) that $\gamma_{1}^{(i)}>0$ for $i=1, \ldots, p$. This is because $\gamma_{1}^{(i)}\left(z-a_{i}\right)$ is the dominating term in the asymptotic expansion. Similarly it follows from $(2.1 b)$ that $\gamma_{-1}^{(\infty)}<0$.

We define linear fractional transformations $s_{1}(z, w), S_{1}(z, w)$ by

$$
s_{1}(z, w)=\frac{-\left(1-\delta_{1}\right)-\alpha_{1}^{-1} \delta_{1} w}{-\alpha_{1}+\left(1-\delta_{1}\right) \beta_{1} z+w}, \quad S_{1}(z, w)=s_{1}(z, w),
$$

where

$$
\begin{aligned}
\delta_{1} & = \begin{cases}1 & \text { if } \gamma_{0}^{(1)}=0, \\
0 & \text { otherwise }\end{cases} \\
\beta_{1} & =-1 / \gamma_{-1}^{(\infty)}, \\
\alpha_{1} & = \begin{cases}\beta_{1} a_{1}+1 / \gamma_{0}^{(1)} & \text { if } \gamma_{0}^{(1)} \neq 0, \\
\text { an arbitrary positive constant } & \text { if } \gamma_{0}^{(1)}=0 .\end{cases}
\end{aligned}
$$

We define a new function $F_{1}(z)$ by

$$
F_{1}(z)=\alpha_{1}-\left(1-\delta_{1}\right) \beta_{1} z-\frac{1}{\delta_{1} \alpha_{1}^{-1}+F(z)} .
$$

Then

$$
F(z)=s_{1}\left(z, F_{1}(z)\right)=S_{1}\left(z, F_{1}(z)\right) .
$$

By (2.1), (2.4), and (2.5) we obtain expansions of the following forms:

(2.7c) $F_{1}(z) \approx \gamma_{1}^{(1,1)}\left(z-a_{1}\right)+\cdots$.

Here $\gamma_{0}^{(\infty, 1)}, \gamma_{-1}^{(i, 1)}$, etc. are new real constants. In the same way new constants will be introduced later.

From $(2.1 b)$ and $(2.4 b)$ it follows that

$$
d_{\infty}=\lim _{\substack{z \rightarrow \infty \\ \operatorname{Re} z=0}}\left(\frac{-1}{z F(z)}\right)=-\frac{1}{\gamma_{-1}^{(\infty)}}=\beta_{1} .
$$


Since $F(z)$ is an $N$-function, it then follows by $(2.5)$ and Lemma 2.1 that

$$
\operatorname{Im} F_{1}(z)=\operatorname{Im}\left(-\frac{1}{\delta_{1} \alpha_{1}^{-1}+F(z)}-\left(1-\delta_{1}\right) \beta_{1} z\right) \geq 0
$$

when $\operatorname{Im}(z)>0$. (Note that $-1 /\left(\alpha_{1}^{-1}+F(z)\right)$ is an $N$-function.) Thus $F_{1}(z)$ is an $N$-function. Therefore, as in the argument preceding the introduction of the transformations $s_{1}(z, w)$ and $S_{1}(z, w)$, we conclude that $\gamma_{-1}^{(\infty, 1)}<0$, $\gamma_{1}^{(1,1)}>0$, and $\gamma_{-1}^{(i, 1)}<0$ or $\gamma_{-1}^{(i, 1)}=0, \gamma_{1}^{(i, 1)}>0$ for $i \neq 1$.

Now let $n \in \mathbf{N}, n \geq 2$. We assume that $N$-functions $F_{1}(z), \ldots, F_{n-1}(z)$ and linear fractional transformations $s_{1}(z, w), \ldots, s_{n-1}(z, w), S_{1}(z, w)$, $\ldots, S_{n-1}(z, w)$ have been defined such that

$$
S_{t}(z, w)=S_{t-1}\left(z, S_{t}(z, w)\right), \quad F(z)=S_{t}\left(z, F_{t}(z)\right)
$$

for $t=1, \ldots, n-1$. As before, we write $r$ for $r_{n}$ and $q$ for $q_{n}$. (For $r=1,2$ the following argument must be modified slightly.) We further assume that expansions for $F_{n-1}(z)$ of the following forms are valid:

$$
\begin{aligned}
& F_{n-1}(z)=\gamma_{1}^{(\infty, n-1)} z+\gamma_{0}^{(\infty, n-1)}+\gamma_{-1}^{(\infty, n-1)}+o\left(z^{-1}\right), \\
& F_{n-1}(z) \approx \gamma_{-1}^{(i, n-1)}\left(z-a_{i}\right)^{-1}+\cdots \quad \text { for } i \neq r-1, r-2, \\
& F_{n-1}(z) \approx \gamma_{0}^{(r-2, n-1)}+\gamma_{1}^{(r-2, n-1)}\left(z-a_{r-2}\right)+\cdots, \\
& F_{n-1}(z) \approx \gamma_{1}^{(r-1, n-1)}\left(z-a_{r-1}\right)+\cdots .
\end{aligned}
$$

We note that all the assumptions are satisfied for $n=2$.

Since $F_{n-1}(z)$ is assumed to be an $N$-function, it follows that $\gamma_{1}^{(\infty, n-1)}>0$ or $\gamma_{1}^{(\infty, n-1)}=0, \gamma_{-1}^{(\infty, n-1)}<0$, and $\gamma_{1}^{(r-2, n-1)}>0, \gamma_{1}^{(r-1, n-1)}>0$, and $\gamma_{-1}^{(i, n-1)}<0$ or $\gamma_{-1}^{(i, n-1)}=0, \gamma_{1}^{(i, n-1)}>0$ for $i \neq r-2, r-1$.

We define linear fractional transformations $s_{n}(z, w), S_{n}(z, w)$ by

$$
\begin{gathered}
s_{n}(z, w)=\frac{\left(\delta_{n}-1\right)+\alpha_{n}^{-1} \delta_{n} w}{\alpha_{n}+\left(\delta_{n}-1\right) \beta_{n} /\left(z-a_{r-1}\right)+w}, \\
S_{n}(z, w)=S_{n-1}\left(z, s_{n}(z, w)\right),
\end{gathered}
$$

where

$$
\alpha_{n}= \begin{cases}\beta_{n} /\left(a_{r}-a_{r-1}\right) & \text { if } \gamma_{-1}^{(r, n-1)} \neq 0, \\ \beta_{n} /\left(a_{r}-a_{r-1}\right)-1 / \gamma_{0}^{(r, n-1)} & \text { if } \gamma_{-1}^{(r, n-1)}=0, \gamma_{0}^{(r, n-1)} \neq 0, \\ \text { an arbitrary positive constant } & \text { if } \gamma_{-1}^{(r, n-1)}=\gamma_{0}^{(r, n-1)}=0 .\end{cases}
$$


We define a new function $F_{n}(z)$ by

$$
F_{n}(z)=-\alpha_{n}+\frac{\left(1-\delta_{n}\right) \beta_{n}}{z-a_{r-1}}-\frac{1}{-\alpha_{n}^{-1} \delta_{n}+F_{n-1}(z)} .
$$

Then

$$
F_{n-1}(z)=s_{n}\left(z, F_{n}(z)\right), \quad F(z)=S_{n}\left(z, F_{n}(z)\right) .
$$

By using (2.11), (2.13), and (2.14), we obtain expansions of the following forms:

$$
\begin{aligned}
& F_{n}(z)=\gamma_{1}^{(\infty, n)} z+\gamma_{0}^{(\infty, n)}+\gamma_{-1}^{(\infty, n)} z^{-1}+o\left(z^{-1}\right), \\
& F_{n}(z) \approx \gamma_{-1}^{(i, n)}\left(z-a_{i}\right)^{-1}+\cdots \text { for } i \neq r, r-1, \\
& F_{n}(z) \approx \gamma_{0}^{(r-1, n)}+\gamma_{1}^{(r-1, n)}\left(z-a_{r-1}\right)+\cdots, \\
& F_{n}(z) \approx \gamma_{1}^{(r, n)}\left(z-a_{r}\right)+\cdots .
\end{aligned}
$$

From $(2.11 \mathrm{~d})$ and $(2.13 \mathrm{~b})$ it follows that

$$
d_{r-1}^{(n-1)}=\lim _{\substack{z \rightarrow a_{r-1} \\ \operatorname{Re}\left(z-a_{r-1}\right)=0}}\left(\frac{-\left(z-a_{r-1}\right)}{-\alpha_{n}^{-1} \delta_{n}+F_{n-1}(z)}\right)=\frac{-1}{\gamma_{1}^{(r-1, n-1)}}=-\beta_{n} .
$$

Since $F_{n-1}(z)$ is an $N$-function, it then follows by (2.14) and Lemma 2.1 that

$$
\operatorname{Im} F_{n}(z)=\operatorname{Im}\left(-\frac{1}{\alpha_{n}^{-1} \delta_{n}+F_{n-1}(z)}+\frac{\left(1-\delta_{n}\right) \beta_{n}}{z-a_{r-1}}\right) \geq 0
$$

when $\operatorname{Im} z \geq 0$. (Note that $-1 /\left(\alpha_{n}^{-1} \delta_{n}+F_{n-1}(z)\right)$ is an $N$-function.) Thus $F_{n}(z)$ is an $N$-function.

We may sum up the results of the foregoing discussion as follows:

Theorem 2.2. Let $F(z)$ be an $N$-function with expansions (2.1). Then the algorithm described above is well defined and determines inductively linear fractional transformations $s_{1}(z, w), \ldots, s_{n}(z, w), \ldots, S_{1}(z, w), \ldots, S_{n}(z, w), \ldots$, and $N$-functions $F_{1}(z), \ldots, F_{n}(z), \ldots$ These are connected by the relations $F_{n-1}(z)=s_{n}\left(z, F_{n}(z)\right), F(z)=S_{n}\left(z, F_{n}(z)\right)$. For each $n$, either $\gamma_{-1}^{(i, n)}<0$ or $\gamma_{-1}^{(i, n)}=0, \gamma_{1}^{(i, n)}>0$ for $i \neq r-1, r-2, \gamma_{1}^{(r-1, n)}>0, \gamma_{1}^{(r, n)}>0$, and either $\gamma_{1}^{(\infty, n)}>0$ or $\gamma_{1}^{(\infty, n)}=0, \gamma_{-1}^{(\infty, n)}<0$.

Now let

$$
\begin{gathered}
\sum_{j=0}^{\infty} \gamma_{j}^{(i)}\left(z-a_{i}\right)^{j}, \quad i=1, \ldots, p \\
\gamma_{-1}^{(\infty)} z^{-1}
\end{gathered}
$$

be given formal power series (with real coefficients). We shall denote this collection by $\phi(z)$. It is readily verified under suitable conditions the algorithm 
we have constructed (with the function $F(z)$ replaced by the series (2.19)) produces linear fractional transformations $s_{n}(z, w), S_{n}(z, w)$ of the form (2.3), (2.12), and collections $\phi_{n}(z)$ of formal power series such that

$$
\phi_{0}(z)=\phi(z), \quad \phi_{n-1}(z)=s_{n}\left(z, \phi_{n}(z)\right) \text { for } n=1,2, \ldots
$$

We note that then also $\phi(z)=S_{n}\left(z, \phi_{n}(z)\right)$. The collections $\phi_{n}(z)$ are of the form

$$
\begin{gathered}
\sum_{j=-1}^{\infty} \gamma_{j}^{(i, n)}\left(z-a_{i}\right)^{j} \quad \text { for } i \neq r, r-1, \\
\sum_{j=0}^{\infty} \gamma_{j}^{(r-1, n)}\left(z-a_{r-1}\right)^{j} \\
\sum_{j=1}^{\infty} \gamma_{j}^{(r, n)}\left(z-a_{r}\right)^{j} \\
\sum_{j=0}^{2} \gamma_{j}^{(\infty, n)} z^{1-j}+o\left(z^{-1}\right)
\end{gathered}
$$

We shall call the collection $\phi(z)$ positive if the following conditions hold:

$$
\gamma_{1}^{(i)}>0 \text { for } i=1, \ldots, p, \quad \gamma_{-1}^{(\infty)}<0
$$

and for $n=1,2, \ldots$,

$\left(\mathbf{P}_{n}\right)$

$$
\left\{\begin{aligned}
& \gamma_{-1}^{(i, n)}<0 \text { or } \gamma_{-1}^{(i, n)}=0, \gamma_{1}^{(i, n)}>0, i=1, \ldots, p, \text { and } \\
& \gamma_{1}^{(\infty, n)}>0 \text { or } \gamma_{1}^{(\infty, n)}=0, \gamma_{-1}^{(\infty, n)}<0 .
\end{aligned}\right.
$$

We observe that if $\left(\mathbf{P}_{0}\right), \ldots,\left(\mathbf{P}_{n-1}\right)$ are satisfied, then $s_{n}(z, w), S_{n}(z, w)$ can be defined.

It follows from the expressions $(2.4 \mathrm{~b}),(2.13 \mathrm{~b})$ that for a positive collection $\phi$, the following inequalities hold:

$$
\beta_{n}>0 \text { for } n=1,2, \ldots \text {. }
$$

We shall call the index $n$ singular if $\delta_{n}=1$ and regular if $\delta_{n}=0$. Thus by (2.4a), 1 is singular iff $\gamma_{0}^{(1)}=0$, and by $(2.13 \mathrm{a}), n$ is singular iff $\gamma_{-1}^{(r, n-1)}=$ $\gamma_{0}^{(r, n-1)}=0$ for $n=2,3, \ldots$.

We have shown above that if the collection $\phi(z)$ consists of asymptotic expansions for a Nevanlinna function $F(z)$, then $\left(\mathrm{P}_{0}\right), \ldots,\left(\mathrm{P}_{n}\right), \ldots$ are automatically satisfied.

We shall in this paper call the algorithm described here the modified Schur algorithm.

\section{RECURRENCE RELATIONS}

Let $\phi(z)$ be a positive collection of formal power series, with associated transformations $s_{n}(z, w), S_{n}(z, w)$. 
Since the $S_{n}(z, w)$ are linear fractional transformations, there exist functions $A_{n}(z), B_{n}(z), C_{n}(z), D_{n}(z), n=1,2, \ldots$, such that

$$
S_{n}(z, w)=\frac{A_{n}(z)+w C_{n}(z)}{B_{n}(z)+w D_{n}(z)} .
$$

For each $n, A_{n}, B_{n}, C_{n}, D_{n}$ are defined up to a common factor $\varphi(z)$.

We call the functions

$$
f_{n}(z)=S_{n}(z, 0)=\frac{A_{n}(z)}{B_{n}(z)}
$$

the approximants for the algorithm. We shall also make use of the functions

$$
g_{n}(z)=S_{n}(z, \infty)=\frac{C_{n}(z)}{D_{n}(z)} .
$$

Since

$$
S_{1}(z, w)=\frac{-\left(1-\delta_{1}\right)-\alpha_{1}^{-1} \delta_{1} w}{-\alpha_{1}+\left(1-\delta_{1}\right) \beta_{1} z+w}
$$

we may set

(3.5) $A_{1}=-\left(1-\delta_{1}\right), \quad B_{1}=-\alpha_{1}+\left(1-\delta_{1}\right) \beta_{1} z, \quad C_{1}=-\alpha_{1}^{-1} \delta_{1}, \quad D_{1}=1$.

From the relation $S_{n}(z, w)=S_{n-1}\left(z, s_{n}(z, w)\right),(2.12)$, and (3.1) we obtain for $n=2,3, \ldots$

$$
\begin{aligned}
& \frac{A_{n}+w C_{n}}{B_{n}+w D_{n}} \\
& \quad=\frac{\left[\left(-\alpha_{n}+\frac{\left(\delta_{n}-1\right) \beta_{n}}{z-a_{r-1}}\right) A_{n-1}+\left(\delta_{n}-1\right) C_{n-1}\right]+\left[A_{n-1}+\alpha_{n}^{-1} \delta_{n} C_{n-1}\right] w}{\left[\left(-\alpha_{n}+\frac{\left(\delta_{n}-1\right)}{z-a_{r-1}}\right) B_{n-1}+\left(\delta_{n}-1\right) D_{n-1}\right]+\left[B_{n-1}+\alpha_{n}^{-1} \delta_{n} D_{n-1}\right] w} .
\end{aligned}
$$

Thus $A_{n}, B_{n}, C_{n}, D_{n}$ satisfy the following recurrence relations for $n=2$, $3, \ldots$ :

$$
\begin{aligned}
& A_{n}=\left(\alpha_{n}+\frac{\left(\delta_{n}-1\right) \beta_{n}}{z-a_{r-1}}\right) A_{n-1}+\left(\delta_{n}-1\right) C_{n-1}, \\
& C_{n}=A_{n-1}+\delta_{n} \alpha_{n}^{-1} C_{n-1}, \\
& B_{n}=\left(\alpha_{n}+\frac{\left(\delta_{n}-1\right) \beta_{n}}{z-a_{r-1}}\right) B_{n-1}+\left(\delta_{n}-1\right) D_{n-1}, \\
& D_{n}=B_{n-1}+\delta_{n} \alpha_{n}^{-1} D_{n-1} .
\end{aligned}
$$

When $n$ is a regular index, these relations can be written as

$$
\begin{aligned}
& A_{n}=\left(\alpha_{n}-\frac{\beta_{n}}{z-a_{r-1}}\right) A_{n-1}-C_{n-1}, \\
& C_{n}=A_{n-1}, \\
& B_{n}=\left(\alpha_{n}-\frac{\beta_{n}}{z-a_{r-1}}\right) B_{n-1}-D_{n-1}, \\
& D_{n}=B_{n-1}
\end{aligned}
$$


while when $n$ is a singular index, the relations may be written as

$$
\begin{array}{ll}
A_{n}=\alpha_{n} A_{n-1}, & C_{n}=A_{n-1}+\alpha_{n}^{-1} C_{n-1}, \\
B_{n}=\alpha_{n} B_{n-1}, & D_{n}=B_{n-1}+\alpha_{n}^{-1} D_{n-1} .
\end{array}
$$

We note that when all $n$ are regular, the algorithm is a continued fraction algorithm. It can be shown that the continued fractions obtained in this way are equivalent to MP-fractions, treated in [7, 8, 24]. (For basic concepts of continued fractions, including the concept of equivalence, see [13].)

For further use we introduce polynomials $N_{n}(z)$ defined as follows:

$$
\begin{aligned}
& N_{0}(z) \equiv 1, \\
& N_{n}(z)=\left(z-a_{1}\right)^{q+1} \cdots\left(z-a_{r}\right)^{q+1}\left(z-a_{r+1}\right)^{q} \cdots\left(z-a_{p}\right)^{q} \\
& \quad \text { for } n=1,2, \ldots .
\end{aligned}
$$

Proposition 3.1. The function $A_{n}, B_{n}, C_{n}, D_{n}$ can be written as

$$
\begin{array}{lll}
A_{n}(z)=\frac{U_{n}(z)}{N_{n-1}(z)}, & B_{n}(z)=\frac{V_{n}(z)}{N_{n-1}(z)}, & n=1,2, \ldots, \\
C_{n}(z)=\frac{X_{n}(z)}{N_{n-2}(z)}, & D_{n}(z)=\frac{Y_{n}(z)}{N_{n-2}(z)}, & n=2,3, \ldots,
\end{array}
$$

where $U_{n}, V_{n}, X_{n}, Y_{n}$ are polynomials, $\operatorname{deg} U_{n} \leq n-1, \operatorname{deg} V_{n} \leq n, \operatorname{deg} X_{n} \leq$ $n-2$, and $\operatorname{deg} Y_{n} \leq n-1$ (except $\left.\operatorname{deg} X_{1}=0\right)$.

Proof. It follows by (3.4) that the result holds for $n=1$, resp. $n=2$, and by use of (3.6)-(3.7) the result is obtained for general $n$ by induction.

For further use it will also be natural to introduce the functions $P_{n}, Q_{n}$ by

$$
P_{n}(z)=\frac{A_{n}(z)}{z-a_{r}}=\frac{U_{n}(z)}{N_{n}(z)}, \quad Q_{n}(z)=\frac{B_{n}(z)}{z-a_{r}}=\frac{V_{n}(z)}{N_{n}(z)} .
$$

We note for further reference that the recurrence relations (3.7) can be written in terms of $U_{n}, V_{n}, X_{n}, Y_{n}$ as follows (for $n=2,3, \ldots$ ):

$$
\begin{gathered}
U_{n}=\left[\alpha_{n}\left(z-a_{r-1}\right)+\left(\delta_{n}-1\right) \beta_{n}\right] U_{n-1} \\
+\left(\delta_{n}-1\right)\left(z-a_{r-2}\right)\left(z-a_{r-1}\right) X_{n-1}, \\
X_{n}=U_{n-1}+\delta_{n} \alpha_{n}^{-1}\left(z-a_{r-2}\right) X_{n-1}, \\
V_{n}=\left[\alpha_{n}\left(z-a_{r-1}\right)+\left(\delta_{n}-1\right) \beta_{n}\right] V_{n-1} \\
+\left(\delta_{n}-1\right)\left(z-a_{r-2}\right)\left(z-a_{r-1}\right) Y_{n-1}, \\
Y_{n}=V_{n-1}+\delta_{n} \alpha_{n}^{-1}\left(z-a_{r-2}\right) Y_{n-1} .
\end{gathered}
$$

Proposition 3.2. Assume that $V_{n}\left(a_{r_{n}}\right) \neq 0$ for all $n$. Then for every $n, V_{n}$ and $Y_{n}$ do not both have a zero at $a_{i}$ for any fixed $i \in\{1, \ldots, p\}$. For every $n$, either $\operatorname{deg} V_{n}=n$ or $\operatorname{deg} Y_{n}=n-1$.

Proof. Let $V_{n}\left(a_{i}\right)=Y_{n}\left(a_{i}\right)=0$. It follows by (3.13) that $V_{n+1}\left(a_{i}\right)=Y_{n+1}\left(a_{i}\right)=$ 0 . By continuing this argument a finite number of steps, we reach the equality $Y_{p q+i}\left(a_{i}\right)=0$ for some $q$. This contradicts the assumption $V_{p+i}\left(a_{i}\right) \neq 0$. 
A similar argument shows that if $\operatorname{deg} V_{n}<n, \operatorname{deg} Y_{n}<n-1$, then we must have $Y_{1} \equiv 0$, which contradicts (3.4).

We present some basic formulas that will be needed later.

Proposition 3.3. The following formulas hold:

(i) $A_{n} D_{n}-B_{n} C_{n}=-1$,

(ii) $A_{n} B_{n-1}-A_{n-1} B_{n}=\left(\delta_{n}-1\right)$,

(iii) $C_{n} D_{n-1}-C_{n-1} D_{n}=-1$,

(iv) $A_{n} D_{n-1}-B_{n} C_{n-1}=-\left(\alpha_{n}+\frac{\left(\delta_{n}-1\right) \beta_{n}}{z-a_{r-1}}\right)$,

(v) $A_{n-1} D_{n}-B_{n-1} C_{n}=-\delta_{n} \alpha_{n}^{-1}$.

Proof. By using (3.7) we obtain $A_{n} D_{n}-B_{n} C_{n}=A_{n-1} D_{n-1}-B_{n-1} C_{n-1}=$ $\cdots=A_{1} D_{1}-B_{1} C_{1}=-1$. Again by using (3.7) and the result of (i) we get $A_{n} B_{n-1}-A_{n-1} B_{n}=\left(\delta_{n}-1\right)\left[A_{n-1} D_{n-1}-B_{n-1} C_{n-1}\right]=1-\delta_{n}$. The formulas (iii), (iv), and (v) are proved analogously.

We next deduce some formulas analogous to the classical Christoffel-Darboux formula and related formulas. (See also [18] for similar formulas, proved within the framework of orthogonal rational functions.)

Proposition 3.4. The following formulas are valid for $n=1,2, \ldots$ :

$$
\begin{aligned}
B_{n}(z) & D_{n}(\zeta)-B_{n}(\zeta) D_{n}(z) \\
= & (z-\zeta) \sum_{m=1}^{n}\left(1-\delta_{m}\right) \beta_{m} Q_{m-1}(z) Q_{m-1}(\zeta), \\
A_{n}(z) & C_{n}(\zeta)-A_{n}(\zeta) C_{n}(z) \\
= & (z-\zeta) \sum_{m=1}^{n}\left(1-\delta_{m}\right) \beta_{m} P_{m-1}(z) P_{m-1}(\zeta), \\
B_{n}(z) & C_{n}(\zeta)-A_{n}(\zeta) D_{n}(z) \\
= & 1+(z-\zeta) \sum_{m=1}^{n}\left(1-\delta_{m}\right) \beta_{n} P_{m-1}(\zeta) Q_{m-1}(z)
\end{aligned}
$$

Proof. By multiplying the difference equation (3.7a) with argument $z$ by the difference equation (3.7b) with argument $\zeta$, and then subtracting the same equation with $z$ and $\zeta$ interchanged, we get for $m=2,3, \ldots$

$$
\begin{aligned}
B_{m}(z) D_{m}(\zeta)-B_{m}(\zeta) D_{m}(z)= & (z-\zeta)\left(1-\delta_{m}\right) \beta_{m} \frac{B_{m-1}(z) B_{m-1}(\zeta)}{\left(z-a_{r-1}\right)\left(\zeta-a_{r-1}\right)} \\
& +B_{m-1}(z) D_{m-1}(\zeta)-B_{m-1}(\zeta) D_{m-1}(\zeta)
\end{aligned}
$$

Furthermore for $m=1$ we get from (3.4)

$$
B_{1}(z) D_{1}(\zeta)-B_{1}(\zeta) D_{1}(z)=\beta_{1}(z-\zeta) .
$$


By adding these equations for $m=1,2, \ldots, n$, taking into account (3.12), we obtain (3.15a).

The proofs of (3.15b) and (3.15c) are similar.

Proposition 3.5. The following formula is valid for $n=1,2, \ldots$ :

$B_{n}(z)-B_{n}(\zeta)=(z-\zeta) \sum_{m=1}^{n}\left(1-\delta_{m}\right) \beta_{m}\left[B_{n}(\zeta) P_{m-1}(\zeta)-A_{n}(\zeta) Q_{m-1}(\zeta)\right] Q_{m-1}(z)$

Proof. Subtraction of (3.15a) multiplied by $A_{n}(\zeta)$ from (3.15c) multiplied by $B_{n}(\zeta)$, with (3.14a) being taken into account, gives (3.18).

Proposition 3.6. The following formula is valid for $n=1,2, \ldots$ :

$$
V_{n}^{\prime}(z) Y_{n}(z)=V_{n}(z) Y_{n}^{\prime}(z)=N_{n-1}(z)^{2} \sum_{m=1}^{n}\left(1-\delta_{m}\right) \beta_{m} Q_{m-1}(z)^{2} .
$$

Proof. Formula (3.15a) may be written as

$$
\begin{aligned}
& V_{n}(z) Y_{n}(\zeta)-V_{n}(\zeta) Y_{n}(z) \\
& \quad=(z-\zeta) N_{n-1}(z) N_{n-1}(\zeta) \sum_{m=1}^{m}\left(1-\delta_{m}\right) \beta_{m} Q_{m-1}(z) Q_{m-1}(\zeta)
\end{aligned}
$$

The left side of this equation may be written as

$$
\left[V_{n}(z) V_{n}(\zeta)\right] Y_{n}(\zeta)-\left[Y_{n}(z)-Y_{n}(\zeta)\right] V_{n}(\zeta)
$$

By dividing (3.20) by $(z-\zeta)$ and letting $\zeta \rightarrow z$, we obtain (3.19).

Corollary 3.7. Assume that $V_{n}\left(a_{r_{n}}\right) \neq 0$ for all $n$. Then for each $a_{i}$ and each $n$, $V_{n}(z)$ has a zero of multiplicity at most one at $a_{i}$. For each $n, \operatorname{deg} V_{n} \geq n-1$. Proof. It follows from (3.13c) and the assumption $V_{m}\left(a_{r_{m}}\right) \neq 0$ that for at least one $m \in\{n-p+1, \ldots, n\}, \delta_{m}=0$. Let $m=p q+i$. For this $m$, the right side of equation (3.19) is different from zero for $z=a_{i}$. Consequently by (3.19), not both $V_{n}\left(a_{i}\right)$ and $V_{n}\left(a_{i}\right)$ can be zero.

The result on the degree can be proved analogously.

We shall see in $\S 4$ that the condition $V_{n}\left(a_{r_{n}}\right) \neq 0$ for all $n$ is always satisfied when the transformations $s_{n}(z, w), S_{n}(z, w)$ and thereby $A_{n}, B_{n}, C_{n}, D_{n}$ are obtained from a positive collection of power series.

\section{Multipoint Padé approximants}

In this section we show that the functions $f_{n}(z)=S_{n}(z, 0)=A_{n}(z) / B_{n}(z)$, or their Taylor series expansions, have certain interpolation properties with respect to the series expansions (2.19) (in particular, to (2.1), when the series originate as expansions of a Nevanlinna function). More precisely, we show that $f_{n}(z)$ is a multipoint Padé approximant for the given series. (For a detailed discussion of multipoint Padé approximants in this setting, we refer the reader to [19].) 
We first give an interpolation result for $f_{n}(z)$ at $a_{r}$ by a direct argument from the construction of the algorithm in $\S 2$. (As before, we write $r$ for $r_{n}$ and $q$ for $q_{n}$.)

Proposition 4.1. For $f_{n}(z)$ the following expansion is valid at $a_{r}$ :

$$
f_{n}(z)=\sum_{j=0}^{2 q} \gamma_{j}^{(r)}\left(z-a_{r}\right)^{j}+O\left(\left(z-a_{r}\right)^{2 q+1}\right) .
$$

Proof. We define $H_{n-1}(z)=s_{n}(z, 0), H_{n-2}(z)=s_{n-1}\left(z, H_{n-1}(z)\right)$, and inductively $H_{n-m}(z)=s_{n-m+1}\left(z, H_{n-m+1}(z)\right)$ for $m \leq n$. Then $H_{0}(z)=f_{n}(z)$. For $H_{n-1}(z)$ we have the expression

$$
H_{n-1}(z)=\frac{\left(\delta_{n}-1\right)}{\alpha_{n}+\beta_{n}\left(\delta_{n}-1\right) /\left(z-a_{r-1}\right)},
$$

while for $F_{n-1}(z)$ we have the expression

$$
F_{n-1}(z) \approx \frac{\left(\delta_{n}-1\right)+\alpha_{n}^{-1} \delta_{n}\left[\gamma_{1}^{(r, n)}\left(z-a_{r}\right)+\cdots\right]}{\left(\delta_{n}-1\right) \beta_{n} /\left(z-a_{r-1}\right)+\gamma_{1}^{(r, n)}\left(z-a_{r}\right)+\cdots} .
$$

It follows that if $\gamma_{-1}^{(r, n-1)}=0$, then

$$
H_{n-1}(z)=\gamma_{0}^{(r, n-1)}+O\left(\left(z-a_{r}\right)\right) .
$$

On the other hand, if $\gamma_{-1}^{(r, n-1)} \neq 0$, then

$$
H_{n-1}(z)=\frac{\delta_{-1}^{(r, n-1)}}{\left(z-a_{r}\right)}+O(1) .
$$

Similarly by repeated comparison of $s_{n-m}\left(z, H_{n-m}(z)\right)$ and $s_{n-m}\left(z, F_{n-m}(z)\right)$ we find that

$$
H_{n-p+2}(z)=\gamma_{0}^{(r, n-p+2)}+O\left(\left(z-a_{r}\right)\right)
$$

if $\gamma_{-1}^{(r, n-p+2)}=0$, while

$$
H_{n-p+2}(z)=\frac{\delta_{-1}^{(r, n-p+2)}}{\left(z-a_{r}\right)}+O(1)
$$

if $\gamma_{-1}^{(r, n-p+2)} \neq 0$.

Now we know that $\gamma_{-1}^{(r, n-p+2)}=0$ (cf. (2.11c) with $n$ replaced by $n-p+$ 1). By comparison of $s_{n-p+2}\left(z, F_{n-p+2}(z)\right)$ and $s_{n-p+2}\left(z, H_{n-p+2}(z)\right)$ we see that the constant term in the expansion of $H_{n-p+1}(z)$ coincides with that for $F_{n-p+1}(z)$ both if $\gamma_{-1}^{(r, n-p+2)}=0$ and if $\gamma_{-1}^{(r, n-p+2)} \neq 0$. Thus, in any case

$$
H_{n-p+1}(z)=\gamma_{0}^{(r, n-p+1)}+O\left(\left(z-a_{r}\right)\right) .
$$

By comparison of $s_{n-p+1}\left(z, F_{n-p+1}(z)\right)$ and $s_{n-p+1}\left(z, H_{n-p+1}(z)\right)$, using (2.13b) with $n$ replaced by $n-p+1$, we then find

$$
H_{n-p}(z)=\gamma_{1}^{(r, n-p)}\left(z-a_{r}\right)+\gamma_{2}^{(r, n-p)}\left(z-a_{r}\right)^{2}+O\left(\left(z-a_{r}\right)^{3}\right) \text {. }
$$


By comparison of $s_{n-p}\left(z, F_{n-p}(z)\right)$ and $s_{n-p}\left(z, H_{n-p}(z)\right)$, using $(2.13 \mathrm{c})$ with $n$ replaced by $n-p$, we find

$$
\begin{aligned}
H_{n-p-1}(z)= & \gamma_{0}^{(r, n-p-1)}+\gamma_{1}^{(r, n-p-1)}\left(z-a_{r}\right) \\
& +\gamma_{2}^{(r, n-p+1)}\left(\left(z-a_{r}\right)^{2}\right)+O\left(\left(z-a_{r}\right)^{3}\right)
\end{aligned}
$$

if $\gamma_{-1}^{(r, n-p-1)}=0$, and

$$
H_{n-p-1}(z)=\gamma_{-1}^{(r, n-p-1)} \frac{1}{z-a_{r}}+\gamma_{0}^{(r, n-p-1)}+O\left(\left(z-a_{r}\right)\right)
$$

if $\gamma_{-1}^{(r, n-1)} \neq 0$.

By repeating these arguments until we reach $H_{0}(z)=f_{n}(z)$, noting that $\gamma_{-1}^{(r)}=0$, we obtain the expansion

$$
f_{n}(z)=\gamma_{0}^{(r)}+\gamma_{1}^{(r)}\left(z-a_{r}\right)+\cdots+\gamma_{2 q}^{(r)}\left(z-a_{r}\right)^{2 q}+O\left(\left(z-a_{r}\right)^{2 q+1}\right) .
$$

Since the functions $f_{n}(z)$ are rational, the asymptotic series for $f_{n}(z)$ are Taylor series.

Proposition 4.2. Let $\phi(z)$ be a positive collection. Then for all $n, V_{n}\left(a_{r}\right) \neq 0$. Proof. It follows directly from (2.4c) and (3.5) that $V_{1}\left(a_{1}\right) \neq 0$. Let $n>1$. We recall that $f_{n}(z)=S_{n}(z, 0)$, while $\phi(z)=S_{n}\left(z, \phi_{n}(z)\right)$. (For brevity we write $\phi(z)$ for the appropriate series.) It follows that

$$
\begin{aligned}
\frac{U_{n}(z)}{V_{n}(z)}-\phi(z) & =\frac{A_{n}}{B_{n}}-\frac{A_{n}+\phi_{n} C_{n}}{B_{n}+\phi_{n} D_{n}}=\frac{U_{n}}{V_{n}}-\frac{U_{n}+\left(z-a_{r-1}\right) X_{n} \phi_{n}}{V_{n}+\left(z-a_{r-1}\right) Y_{n} \phi_{n}} \\
& =\frac{\left(z-a_{r-1}\right) \phi_{n}\left[U_{n} Y_{n}-X_{n} V_{n}\right]}{V_{n}\left[U_{n}+\left(z-a_{r-1}\right) \phi_{n} Y_{n}\right]} .
\end{aligned}
$$

By (4.1) the left side of (4.12) has expansion of the form $f_{n}(z)-\phi(z) \approx$ $\delta_{2 q+1}^{(r)}\left(z-a_{r}\right)^{2 q+1}+\cdots$. The numerator of the right side of (4.12) has expansion of the form $-\phi_{n}(z) N_{n-1}(z)^{2} \approx \gamma_{1}^{(r, n)}\left(z-a_{r}\right)^{2 q+1}+\cdots$. For (4.12) to hold it is thus necessary that $V_{n}\left(a_{r}\right) \neq 0$.

We note that in exactly the same way as (4.12) we get

$$
\frac{X_{n}(z)}{Y_{n}(z)}-\phi(z)=\frac{\left[V_{n} X_{n}-U_{n} Y_{n}\right]}{Y_{n}\left[V_{n}+\left(z-a_{r-1}\right) \phi_{n} Y_{n}\right]} .
$$

We now state the interpolation theorem for the approximants $f_{n}(z)$ with respect to $F(z)$, or to the series expansions (2.19).

Theorem 4.3. The approximant $f_{n}(z)=U_{n}(z) / V_{n}(z)$ has the following interpolation properties: 
(4.14) $\frac{U_{n}(z)}{V_{n}(z)}=\sum_{j=0}^{2 q+1} \gamma_{j}^{(i)}\left(z-a_{i}\right)^{j}+O\left(\left(z-a_{i}\right)^{2 q+2}\right)$ if $i<r, V_{n}\left(a_{i}\right) \neq 0$,

(4.15) $\frac{U_{n}(z)}{V_{n}(z)}=\sum_{j=0}^{2 q} \gamma_{j}^{(r)}\left(z-a_{r}\right)^{j}+O\left(\left(z-a_{r}\right)^{2 q+1}\right)$,

(4.16) $\frac{U_{n}(z)}{V_{n}(z)}=\sum_{j=0}^{2 q-1} \gamma_{j}^{(i)}\left(z-a_{i}\right)^{j}+O\left(\left(z-a_{i}\right)^{2 q}\right)$ if $i>r, V_{n}\left(a_{i}\right) \neq 0$,

(4.17) $\frac{U_{n}(z)}{V_{n}(z)}=-\gamma_{-1}^{(\infty)} z^{-1}+o\left(z^{-1}\right)$ if $\operatorname{deg} V_{n}=n$.

In general, the approximant has the following (weak) multipoint Pade approximation property:

$$
\begin{aligned}
& U_{n}(z)-V_{n}(z) \sum_{j=0}^{2 q+1} \gamma_{j}^{(i)}\left(z-a_{i}\right)^{j}=O\left(\left(z-a_{i}\right)^{2 q+2}\right) \quad \text { if } i<r, \\
& U_{n}(z)-V_{n}(z) \sum_{j=0}^{2 q} \gamma_{j}^{(r)}\left(z-a_{r}\right)^{j}=O\left(\left(z-a_{r}\right)^{2 q+1}\right), \\
& U_{n}(z)-V_{n}(z) \sum_{j=0}^{2 q-1} \gamma_{j}^{(i)}\left(z-a_{i}\right)^{j}=O\left(\left(z-a_{i}\right)^{2 q}\right) \quad \text { if } i>r, \\
& U_{n}(z)-V_{n}(z)\left(-\gamma_{-1}^{(\infty)} z^{-1}\right)=o\left(z^{-1}\right) .
\end{aligned}
$$

Proof. Formula (4.15) follows immediately from Proposition 4.1.

Let, e.g., $i<r$. For each of the numbers $m \in\{p q+i+1, \ldots, n-1\}$ let $Z_{m}$ and $W_{m}$ denote $X_{m}$ and $Y_{m}$, or $U_{m}$ and $V_{m}$, such that $W_{m}\left(a_{i}\right) \neq 0$ (cf. Proposition 3.2). According to Proposition 3.3 and (3.11) we may write for $m \in\{p q+i+2, \ldots, n-1\}$ :

$$
\frac{Z_{m}}{W_{m}}-\frac{Z_{m-1}}{W_{m-1}}=\frac{Z_{m} W_{m-1}-Z_{m-1} W_{m}}{W_{m} W_{m-1}}=\frac{c(z) N_{m-1}(z) N_{m-2}(z)}{W_{m}(z) W_{m-1}(z)},
$$

where $c(z)$ is a constant or a simple rational function without a pole at $a_{i}$. Thus,

$$
\frac{Z_{m}}{W_{m}}-\frac{Z_{m-1}}{W_{m-1}}=O\left(\left(z-a_{i}\right)^{2 q+2}\right) \text { for } m=p q+i+2, \ldots, n-1 .
$$

Similarly we conclude that

$$
\frac{U_{n}}{V_{n}}-\frac{Z_{n-1}}{W_{n-1}}=\frac{c(z) N_{n-1}(z) N_{n-2}(z)}{V_{n}(z) W_{n-1}(z)},
$$

where $c(z)$ is as above. From this we conclude that we may write

$$
\frac{U_{n}(z)}{V_{n}(z)}-\frac{Z_{n-1}(z)}{W_{n-1}(z)}=\frac{d\left(z-a_{i}\right)^{2 a+2}+\cdots}{V_{n}(z)} \text {. }
$$


From (4.12)-(4.13) together with (3.11) and Proposition 3.3 we obtain

$$
\frac{Z_{p q+i+1}(z)}{W_{p q+i+1}(z)}-\phi(z)=\frac{\omega(z) N_{p q+i}(z)^{2}}{W_{p q+i+1}(z)\left[V_{p q+i+1}(z)+\left(z-a_{i}\right) Y_{p q+i+1}(z)\right]},
$$

where $\omega(z)$ is regular at $a_{i}$. Thus,

$$
\frac{Z_{p q+i+1}(z)}{W_{p q+i+1}(z)}-\phi(z)=O\left(\left(z-a_{i}\right)^{2 q+2}\right) .
$$

We may write

$$
\begin{aligned}
f_{n}(z)-\phi(z)= & \left(\frac{U_{n}(z)}{V_{n}(z)}-\frac{Z_{n-1}(z)}{W_{n-1}(z)}\right)+\left(\frac{Z_{n-1}(z)}{W_{n-1}(z)}-\frac{Z_{n-2}(z)}{W_{n-2}(z)}\right) \\
& +\cdots+\left(\frac{Z_{p q+i+2}(z)}{W_{p q+i+2}(z)}-\frac{Z_{p q+i+1}(z)}{W_{p q+i+2}(z)}\right)+\left(\frac{Z_{p q+i+1}(z)}{W_{p q+i+1}(z)}-\phi(z)\right) .
\end{aligned}
$$

It follows by (4.23), (4.25), and (4.27) that we may write

$$
f_{n}(z)-\phi(z)=\frac{e\left(z-a_{i}\right)^{2 q+2}}{V_{n}(z)}+O\left(\left(z-a_{i}\right)^{2 q+2}\right) .
$$

Thus if $V_{n}\left(a_{i}\right) \neq 0$ we get $f_{n}(z)-\phi(z)=O\left(\left(z-a_{i}\right)^{2 q+2}\right)$, and in all cases $U_{n}(z)-V_{n}(z) \cdot \phi(z)=O\left(\left(z-a_{i}\right)^{2 q+2}\right)$. This implies (4.14) and (4.18).

Similarly we prove the formula

$$
f_{n}(z)-\phi(z)=\frac{e\left(z-a_{i}\right)^{2 q+2}+\cdots}{V_{n}(z)}+O\left(\left(z-a_{i}\right)^{2 q}\right)
$$

in the case that $i>r$. Thus if $V_{n}\left(a_{i}\right) \neq 0$ we get $f_{n}(z)-\phi(z)=O\left(\left(z-a_{i}\right)^{2 q}\right)$, and in all cases $U_{n}(z)-V_{n}(z) \cdot \phi(z)=O\left(\left(z-a_{i}\right)^{2 q}\right)$. This implies (4.16) and (4.20).

Finally a completely analogous argument shows that $f_{n}(z)-\phi(z)=o\left(z^{-1}\right)$ if $\operatorname{deg} V_{n}=n$ and, in general, $U_{n}(z)-V_{n}(z) \cdot \phi(z)=o\left(z^{n-1}\right)$. (Here it has to be used that $f_{1}(z)-\phi(z)=\gamma_{-1}^{(\infty)} z^{-1}+o\left(z^{-1}\right)$, which follows immediately from the construction of $s_{1}(z, w)$.) This implies (4.17) and (4.21). This completes the proof.

Remark 4.4. It easily follows that if $V_{n}\left(a_{i}\right)=0$, and hence $Y_{n}\left(a_{i}\right) \neq 0$, then (4.14)-(4.16) hold with $U_{n}(z) / V_{n}(z)$ replaced by $X_{n}(z) / Y_{n}(z)$ and $r$ by $r-1$. Similarly (4.17) holds with $U_{n}(z) / V_{n}(z)$ replaced by $X_{n}(z) / Y_{n}(z)$ if $\operatorname{deg} V_{n}<$ $n$, and hence $\operatorname{deg} Y_{n}=n-1$.

Remark 4.5. From Theorem 4.3 and the recurrence relations (3.13) it can be seen that among the $p q$ first indices there are at least $q$ regular ones (cf. the proof of Corollary 3.7). 
We define the generalized approximants for the algorithm by

$$
f_{n}(z, \tau)=\frac{A_{n}(z)+\tau C_{n}(z)}{B_{n}(z)+\tau D_{n}(z)}, \quad \tau \in \mathbf{R} .
$$

We may clearly also write

$$
f_{n}(z, \tau)=\frac{U_{n}(z)+\tau\left(z-a_{r-1}\right) X_{n}(z)}{V_{n}(z)+\tau\left(z-a_{r-1}\right) Y_{n}(z)} .
$$

Proposition 4.6. For every $n$ and every $\tau \in \mathbf{R}$ the following formulas hold (except possibly for one value for $\tau$ in each case):

$$
\begin{aligned}
& f_{n}(z, \tau)=\sum_{j=0}^{2 q+1} \gamma_{j}^{(i)}\left(z-a_{i}\right)^{j}+O\left(\left(z-a_{i}\right)^{2 q+2}\right) \quad \text { if } i<r-1, \\
& f_{n}(z, \tau)=\sum_{j=0}^{2 q-1} \gamma_{j}^{(i)}\left(z-a_{i}\right)^{j}+O\left(\left(z-a_{i}\right)^{2 q}\right) \quad \text { if } i \geq r-1, \\
& f_{n}(z, \tau)=\gamma_{-1}^{(\infty)} z^{-1}+o\left(z^{-1}\right) \quad \text { if } \operatorname{deg} V_{n}=n .
\end{aligned}
$$

Proof. We may write

$$
\begin{aligned}
f_{n}(z, \tau)-\frac{U_{n}(z)}{V_{n}(z)} & =\frac{A_{n}(z)+\tau C_{n}(z)}{B_{n}(z)+\tau D_{n}(z)}-\frac{A_{n}(z)}{B_{n}(z)} \\
& =\frac{\tau N_{n-1}(z)^{2}}{V_{n}(z)\left[V_{n}(z)+\tau\left(z-a_{r-1}\right) Y_{n}(z)\right]}, \\
f_{n}(z, \tau)-\frac{X_{n}(z)}{Y_{n}(z)} & =\frac{A_{n}(z)+\tau C_{n}(z)}{B_{n}(z)+\tau D_{n}(z)}-\frac{C_{n}(z)}{D_{n}(z)} \\
& =\frac{N_{n-1}(z) N_{n-2}(z)}{Y_{n}(z)\left[U_{n}(z)+\tau\left(z-a_{r-1}\right) Y_{n}(z)\right]} .
\end{aligned}
$$

We note that since $V_{n}(z)$ and $Y_{n}(z)$ are not both zero for any $a_{i}$, then $\left[V_{n}(z)+\right.$ $\tau\left(z-a_{r-1}\right) Y_{n}(z)$ ] has no zero except possibly for one value of $\tau$, when $i \neq r-1$. When $i=r-1,\left[V_{n}(z)+\tau\left(z-a_{r-1}\right) Y_{n}(z)\right]$ may, except for one value of $\tau$, have a zero of multiplicity at most one (cf. Corollary 3.7). This together with (4.36) and Theorem 4.3 or (4.37) and Remark 4.4 gives (4.33)-(4.34). The proof of (4.35) is analogous.

\section{MAPPING PROPERTIES}

In this section we discuss mapping properties of the linear fractional transformations $s_{n}(z, w), S_{n}(z, w)$ (see also [8]). Let $z \in H_{+}^{0}$. Each of the linear fractional transformations $w \rightarrow s_{n}(z, w)$ maps the closed upper half-plane $H_{+}$onto a disk $\delta_{n}(z)$ (possibly a half-plane), and each of the linear fractional transformations $w \rightarrow S_{n}(z, w)$ maps $H_{+}$onto a disk (possibly a half-plane) $\Delta_{n}(z)$. 
Propostiion 5.1. The following inclusions hold for all $z \in H_{+}^{0}$ :

(A) $\Delta_{1}(z) \subset H_{+}$.

(B) $\Delta_{n}(z) \subset \Delta_{n-1}(z)$ for $n=2,3, \ldots$.

Proof. Since $\beta_{n}>0$ for $n=1,2, \ldots$, it is readily verified that $s_{n}(z, w) \in$ $H_{+}$for all $n$ and all $w \in H_{+}$. This means that $\delta_{n}(z) \subset H_{+}$. It follows that $\Delta_{1}(z)=\delta_{1}(z) \subset H_{+}$and $\Delta_{n}(z)=S_{n}\left(z, H_{+}\right)=S_{n-1}\left(z, \delta_{n}(z)\right) \subset S_{n-1}\left(z, H_{+}\right)$ $=\Delta_{n-1}(z)$.

We recall that the mapping $w \rightarrow S_{n}(z, w)$ is described by the formula

$$
S_{n}(z, w)=\frac{A_{n}(z)+w C_{n}(z)}{B_{n}(z)+w D_{n}(z)} .
$$

$S_{n}(z, \tau)$ traces the boundary $\partial \Delta_{n}(z)$ when $\tau$ traces the real axis (plus $\infty$ ). For convenience we shall in the following write $h_{m-1}$ for $\sqrt{\left(1-\delta_{m}\right) \beta_{m}}$. (Recall that $\beta_{m}>0, \delta_{m}=0$ or $\delta_{m}=1$.)

Proposition 5.2. For every $n=1,2,3,4, \ldots$, the radius $\rho_{n}(z)$ of the disk $\Delta_{n}(z)$ is given by the formula

$$
\frac{1}{\rho_{n}(z)}=|z-\bar{z}| \cdot \sum_{m=1}^{n} h_{m-1}^{2}\left|Q_{m-1}(z)\right|^{2} .
$$

Proof. By standard results on linear fractional transformations it follows (using formula (5.1)) that $\rho_{n}(z)$ can be expressed as

$$
\rho_{n}(z)=\left|\frac{A_{n}(z) D_{n}(z)-C_{n}(z) B_{n}(z)}{B_{n}(z) D_{n}(\bar{z})-C_{n}(z) B_{n}(\bar{z})}\right| .
$$

(We recall that all the coefficients in $A_{n}(z), B_{n}(z), C_{n}(z), D_{n}(z)$ are real.) From (3.14a) we get

$$
\left|A_{n}(z) D_{n}(z)-C_{n}(z) B_{n}(z)\right|=1 .
$$

From $(3.15 \mathrm{a})$ we get (setting $\zeta=\bar{z}$ )

$$
\left|B_{n}(z) D_{n}(\bar{z})-D_{n}(z) B_{n}(\bar{z})\right|=|z-\bar{z}| \cdot \sum_{m=1}^{n} h_{m-1}^{2}\left|Q_{m-1}(z)\right|^{2} .
$$

Formula (5.2) now immediately follows.

We write $\Delta(z)$ for the intersection $\bigcap_{n=1}^{\infty} \Delta_{n}(z)$. Then (by Proposition 5.2) $\Delta(z)$ is a closed disk or reduces to a single point.

Proposition 5.3. The following statements are equivalent:

(A) $\Delta(z)$ reduces to a single point.

(B) $\sum_{m=1}^{\infty} h_{m-1}^{2}\left|B_{m-1}(z)\right|^{2}=\infty$.

(C) $\sum_{m=1}^{\infty} h_{m-1}^{2}\left|A_{m-1}(z)\right|^{2}=\infty$. 
Proof. The radius $\rho(z)$ of $\Delta(z)$ is obviously given by $\rho(z)=\lim _{n \rightarrow \infty} \rho_{n}(z)$, hence

$$
\frac{1}{\rho(z)}=|z-\bar{z}| \sum_{m=1}^{\infty} h_{m-1}^{2}\left|Q_{m-1}(z)\right|^{2} .
$$

Clearly $\sum_{m=1}^{\infty} h_{m-1}^{2}\left|Q_{m-1}(z)\right|^{2}=\infty$ iff $\sum_{m=1}^{\infty} h_{m-1}^{2}\left|B_{m-1}(z)\right|^{2}=\infty$, since $\min \left(\left|z-a_{i}\right|, \ldots,\left|z-a_{p}\right|\right)>0$ for a given $z \in H_{+}^{0}$. Thus (A) and (B) are equivalent.

The transformation $w \rightarrow \frac{1}{S_{n}(z, w)}$ gives rise to a sequence $\left\{\Pi_{n}(z)\right\}$ of nested disks, where

$$
\Pi_{n}(z)=\left\{\zeta=1 / S_{n}(z, w): w \in H_{+}^{0}\right\} .
$$

The intersection $\Pi(z)$ is a disk when $\Delta(z)$ is a disk and reduces to a single point when $\Delta(z)$ reduces to a single point. The transformation $w \rightarrow 1 / S_{n}(z, w)$ can be written as $w \rightarrow\left(B_{n}(z)+w D_{n}(z)\right) /\left(A_{n}(z)+w C_{n}(z)\right)$. The radius $\pi_{n}(z)$ of $\Pi_{n}(z)$ is given by

$$
\pi_{n}(z)=\left|\frac{B_{n}(z) C_{n}(z)-A_{n}(z) D_{n}(z)}{A_{(z)} C_{n}(\bar{z})-C_{n}(z) A_{n}(\bar{z})}\right| .
$$

Just as we obtained (5.2) from (3.14a), (3.15a), and (5.3), we obtain from $(3.14 a),(3.15 b)$ and $(5.6)$ the formula

$$
\frac{1}{\pi_{n}(z)}=|z-\bar{z}| \cdot \sum_{m=1}^{n} h_{m-1}^{2}\left|P_{m-1}(z)\right|^{2} .
$$

Clearly $\sum_{m=1}^{\infty} h_{m-1}^{2}\left|P_{m-1}(z)\right|^{2}=\infty$ iff $\sum_{m=1}^{\infty} h_{m-1}^{2}\left|A_{m-1}(z)\right|^{2}=\infty$. Thus $\Pi(z)$, and hence $\Delta(z)$, reduces to a single point iff $\sum_{m=1}^{\infty} h_{m-1}^{2}\left|A_{m-1}(z)\right|^{2}=\infty$, i.e., (A) and (C) are equivalent.

Theorem 5.4. If $\Delta(z)$ reduces to a single point for some $z \in H_{+}^{0}$, then $\Delta(z)$ reduces to a single point for every $z \in H_{+}^{0}$.

Proof. The argument we give builds on a lemma of Perron [26], and is similar to the one given in [10] for APT-fractions.

It follows from [26] that when $b_{n}$ is given recursively by

$$
b_{n}=\sum_{i=0}^{n-1} a_{n, i} b_{i}+c_{n}, \quad n=1,2,3, \ldots,
$$

then

$$
\ln \left(1+\sum_{i=1}^{n}\left|b_{i}\right|^{2}\right) \leq \sum_{i=1}^{n-1} \sum_{j=0}^{i-1}\left|a_{i j}\right|^{2}+\sum_{i=1}^{n-1}\left|c_{i}\right|^{2}
$$

Set

$$
\begin{gathered}
b_{n}=h_{n} B_{n}(z), \quad c_{n}=h_{n} B_{n}(\zeta) \\
a_{n, i}=h_{i} h_{n}\left[B_{n}(\zeta) P_{i}(\zeta)-A_{n}(\zeta) Q_{i}(\zeta)\right] \frac{(\zeta-z)}{\left(z-a_{r_{i}}\right)} .
\end{gathered}
$$


Then it follows from Proposition 3.5 that $(5.8)$ is satisfied for $n=1,2, \ldots$. Hence from (5.9) we get

$$
\begin{aligned}
\ln (1 & \left.+\sum_{i=1}^{n-1}\left|B_{i}(z)\right|^{2}\right) \\
& \leq \sum_{i=1}^{n-1}\left|B_{i}(\zeta)\right|^{2}+|\zeta-z|^{2} \sum_{i=1}^{n-1} \sum_{j=0}^{i-1} h_{i}^{2} \frac{\left|\left[B_{i}(\zeta) A_{j}(\zeta)-A_{i}(\zeta) B_{j}(\zeta)\right]\right|^{2}}{\left|z-a_{n_{j}}\right|^{2}\left|\zeta-a_{n_{j}}\right|^{2}}
\end{aligned}
$$

Set $d=\min \left\{\left|z-a_{1}\right|, \ldots,\left|z-a_{p}\right|,\left|\zeta-a_{1}\right|, \ldots,\left|\zeta-a_{p}\right|\right\}$. By elementary inequalities we get

$$
\begin{gathered}
\sum_{i=1}^{n-1} \sum_{j=0}^{i-1} \frac{h_{j}^{2} h_{j}^{2}}{\left|\zeta-a_{r_{j}}\right|^{2}\left|\zeta-a_{r_{j}}\right|^{2}}\left|B_{i}(\zeta) A_{j}(\zeta)-A_{i}(\zeta) B_{j}(\zeta)\right|^{2} \\
\leq \frac{2}{d^{4}}\left(\sum_{i=1}^{n-1} h_{i}^{2}\left|A_{i}(\zeta)\right|^{2}\right) \cdot\left(\sum_{i=1}^{n-1} h_{i}^{2}\left|B_{i}(\zeta)\right|^{2}\right)
\end{gathered}
$$

Substitution of (5.13) in (5.12) leads to

$$
\begin{aligned}
\ln (1 & \left.+\sum_{i=1}^{n-1} h_{i}^{2}\left|B_{i}(z)\right|^{2}\right) \\
& \leq \sum_{i=1}^{n-1} h_{i}^{2}\left|B_{i}(\zeta)\right|^{2}+\frac{2|\zeta-z|^{2}}{d^{4}}\left(\sum_{i=1}^{n-1} h_{i}^{2}\left|A_{i}(\zeta)\right|^{2}\right) \cdot\left(\sum_{i=1}^{n-1} h_{i}^{2}\left|B_{i}(\zeta)\right|^{2}\right) .
\end{aligned}
$$

Now assume that $\Delta(\zeta)$ is a disk for some $\zeta$. Let $z \in H_{+}^{0}$ be arbitrary. Then by Proposition 5.3, the right-hand side of (5.14) has a finite upper bound $M$ independent of $n$, and consequently $\sum_{i=1}^{\infty} h_{i}^{2}\left|B_{i}(z)\right|^{2}<\infty$. Then by Proposition 5.3, $\Delta(z)$ is a disk.

In view of Theorem 5.4 we may use the terms limit point situation (when $\Delta(z)$ reduces to a single point) and limit circle situation (when $\Delta(z)$ is a disk) without reference to a specified point $z$.

For further use we shall also give a formula for the center $\sigma_{n}(z)$ of the disk $\Delta_{n}(z)$.

Proposition 5.5. For every $n=1,2, \ldots$, the center $\sigma_{n}(z)$ of the disk $\Delta_{n}(z)$ is given by the formula

$$
\sigma_{n}(z)=\frac{1+(\bar{z}-z) \sum_{m=1}^{n} h_{m-1}^{2} P_{m-1}(z) Q_{m-1}(\bar{z})}{(\bar{z}-z) \sum_{m=1}^{n} h_{m-1}^{2}\left|Q_{m-1}(z)\right|^{2}} .
$$

Proof. By standard results on linear fractional transformations (using (5.1)) it follows that

$$
\sigma_{n}(z)=\frac{C_{n}(z) B_{n}(\bar{z})-A_{n}(z) D_{n}(\bar{z})}{B_{n}(z) D_{n}(\bar{z})-D_{n}(z) B_{n}(\bar{z})}
$$


(Recall that all the coefficients in $A_{n}(z), B_{n}(z), C_{n}(z), D_{n}(z)$ are real.) From (3.15a) we conclude by setting $\zeta=\bar{z}$ that the denominator of (5.16) equals $(\bar{z}-z) \sum_{m=1}^{n} h_{m-1}^{2}\left|Q_{m-1}(z)\right|^{2}$. Similarly we conclude from $(3.15 \mathrm{c})$ that the numerator of $(5.16)$ equals $1+(\bar{z}+z) \sum_{m=1}^{n} h_{m-1}^{2} P_{m-1} Q_{m-1}(\bar{z})$.

\section{ASYMPTOTIC EXPANSIONS AND NEVANLINNA FUNCTIONS}

In this section we discuss the solution of the modified Pick-Nevanlinna problem.

Theorem 6.1. Let $F(z)$ be a Nevanlinna function with the asymptotic expansions (2.1a)-(2.1b). Let $\Delta(z)$ be the disk (or point) associated with $F(z)$ through the modified Schur algorithm, as described in $\S \S 2$ and 5. Then $F(z) \in \Delta(z)$ for every $z \in H_{+}^{0}$.

Proof. For every $z \in H_{+}^{0}$ we have $F(z)=S_{n}\left(z, F_{n}(z)\right)$ (cf. (2.15)), where $F_{n}(z)$ are the tail functions defined by the algorithm. The functions $F_{n}(z)$ are Nevanlinna functions. Therefore $F_{n}(z) \in H_{+}$and so $S_{n}\left(z, F_{n}(z)\right) \in \Delta_{n}(z)$ for all $z \in H_{+}^{0}$. Thus $F(z) \in \Delta_{n}(z)$ for all $n$, i.e., $F(z) \in \Delta(z)$.

Theorem 6.2. Let a positive collection of power series (2.19) be given. Let $F(z)$ be a function which is analytic in $H_{+}^{0}$, and such that $F(z) \in \Delta(z)$ for all $z \in H_{+}^{0}$, where $\Delta(z)$ are the disks or points associated with the series through the modified Schur algorithm as described in $\S \S 2$ and 5. Then $F(z)$ is a Nevanlinna function, and $F(z)$ has the asymptotic expansions (2.19a).

Proof. That $F(z)$ is a Nevanlinna function follows from the fact that $\Delta(z) \subset$ $H_{+}$for all $z \in H_{+}^{0}$.

Let $\delta>0$ be given, and let $r \in\{1, \ldots, p\}$ be fixed. Then for every $z \in R_{r, \delta}$ we have $\left|\operatorname{Im}\left(z-a_{r}\right) /\left(z-a_{r}\right)\right|>\delta$, hence $\left|(z-\bar{z}) /\left(z-a_{r}\right)\right|>\delta$. Let $N$ be an arbitrary natural number, and let $n$ be a natural number such that $r_{n}=r$ and $2 q-2 \geq N$, where $q=q_{n}$. We see that when $\left\{\sum_{m=1}^{n+1} h_{m-1}^{2} Q_{m-1}(z)^{2}\right\}^{-1}$ is expanded in power series in terms of $\left(z-a_{r}\right)$, the leading term will be of the form $k\left(z-a_{r}\right)^{2 q}$ (recall that $\left.V_{n}\left(a_{r}\right) \neq 0\right)$. Thus there exists a constant $c_{r}$ such that for $z \in R_{r, \delta}$ and $z-a_{r}$ sufficiently small, we have

$$
\rho_{n+1}(z) \leq c_{r}\left|z-a_{r}\right|^{2 q-1} \text {. }
$$

(Recall that $\left|\left(z-a_{r}\right) /(z-\bar{z})\right| \leq \delta$.) We have $f_{n+1}(z, \tau) \in \Delta_{n+1}(z)$, while $F(z) \in \Delta(z) \subset \Delta_{n+1}(z)$. It follows that

$$
\left|F(z)-f_{n+1}(z, \tau)\right| \leq 2 c_{r}\left|z-a_{r}\right|^{2 q-1} .
$$

It follows from (4.16) and Remark 4.4 (with $n$ replaced by $n+1$ ) that

$$
f_{n+1}(z, \tau)=\sum_{j=0}^{2 q+1} \gamma_{j}^{(r)}\left(z-a_{r}\right)^{j}+O\left(\left(z-a_{r}\right)^{2 q+2}\right) \text { for } \tau=0 \text { or for } \tau=\infty
$$


The function $f_{n+1}(z, \tau)$ is a rational function, and it has therefore a convergent Taylor series in a neighborhood of $a_{r}$. (Note that $f_{n}(z, 0)$ is analytic at $a_{r}$ if $V_{n+1}\left(a_{r}\right) \neq 0$, and $f_{n}(z, \infty)$ is analytic at $a_{r}$ if $Y_{n+1}\left(a_{r}\right) \neq 0$.) Consequently there exists a constant $d_{r}$ such that

$$
\left|f_{n+1}(t, \tau)-\sum_{j=0}^{2 q+1} \gamma_{j}^{(r)}\left(z-a_{r}\right)^{j}\right| \leq d_{r}\left|z-a_{r}\right|^{2 q+2} \text { for } \tau=0 \text { or } \tau=\infty,
$$

when $z \in R_{r, \delta}, z-a_{r}$ sufficiently small. Combining (6.2) and (6.4) we see that there exists a constant $k_{r}$ such that

$$
\left|F(z)-\sum_{j=0}^{2 q+1} \gamma_{j}^{(r)}\left(z-a_{r}\right)^{j}\right| \leq k_{r}\left|z-a_{r}\right|^{2 q-1}
$$

when $z \in R_{r, \delta}, z-a_{r}$ sufficiently small. Since $N \leq 2 q-2$, it follows that there exists a constant $K_{r}$ such that

$$
\left|F(z)-\sum_{j=0}^{N} \gamma_{j}^{(r)}\left(z-a_{r}\right)^{j}\right| \leq K_{r}\left|z-a_{r}\right|^{N+1}
$$

when $z \in R_{r, \delta}, z-a_{r}$ sufficiently small. This means that

$$
F(z) \approx \sum_{j=0}^{\infty} \gamma_{j}^{(r)}\left(z-a_{r}\right)^{j} .
$$

Theorem 6.2 together with Theorem 6.1 characterize the solutions of the modified Pick-Nevanlinna problem for a positive collection (2.19) of power series. $(F(z)$ is a solution iff $(2.1 b)$ holds and $F(z) \in \Delta(z)$ for all $z$.) However, Theorem 6.2 does not in itself insure that there exist any solutions. We now turn to this problem.

Theorem 6.3. Let a positive collection of power series (2.19) be given. Let $z_{0}$ be an arbitrary point in $H_{+}^{0}$, and let $\omega_{0}$ be an arbitrary point on $\partial \Delta\left(z_{0}\right)$. Then for each $n$ there exists a $\tau_{n} \in \mathbf{R}$ such that a subsequence of $\left\{f_{n}\left(z, \tau_{n}\right)\right\}$ converges on $H_{+}^{0}$ (uniformly on compact subsets) to a function $F(z)$ which has the asymptotic expansions (2.19) and which satisfies $F\left(z_{0}\right)=\omega_{0}$.

Proof. Let $\delta>0, \eta>0$ be given, and set $S_{\delta, \eta}=\left\{z \in R_{\delta}: \operatorname{Im} z>\eta\right\}$. For $z \in R_{\delta}$ we have $|z-\bar{z}|=|2 \operatorname{Im} z| \geq 2|z| \sin \delta$. Let $m$ be the smallest regular index. In view of (5.2) we have $\rho_{m}(z)=1 / h_{m-1}^{2}|z-\bar{z}|$, hence there exists a constant $A$ such that $\left|\rho_{1}(z)\right| \leq A /|z|$ for $z \in R_{\delta}$. Similarly, in view of (5.2) we have $\sigma_{m}(z)=1 / h_{m-1}^{2}|z-\bar{z}|$, hence also $\left|\sigma_{1}(z)\right| \leq A /|z|$ for $z \in R_{\delta}$. Since $f_{n}(z, \tau) \in \partial \Delta_{n}(z)$, we then have

$$
\left|z f_{n}(z, \tau)\right| \leq 2 A
$$

for every generalized approximant $f_{n}(z, \tau), z \in R_{\delta}$. 
For each $n$ we can choose a $\tau_{n} \in \mathbf{R}$ such that $f_{n}\left(z_{0}, \tau_{n}\right)=S_{n}\left(z_{0}, \tau_{n}\right)=\omega_{n}$, where $\omega_{n} \rightarrow \omega_{0}$ as $n \rightarrow \infty$. It is easily seen that every compact subset of $H_{+}^{0}$ is contained in a set $R_{\delta}$. It follows by using standard results on compactness of families of analytic functions (see, e.g., $[9,13])$ that $\left\{f_{n}\left(z, \tau_{n}\right)\right\}$ contains a subsequence $\left\{f_{n_{k}}\left(z, \tau_{n_{k}}\right)\right\}$ such that $\operatorname{deg} V_{n_{k}}=n_{k}$ or $\operatorname{deg} Y_{n_{k}}=n_{k}-1$, which converges on $H_{+}^{0}$ to a function $F(z)$ which is analytic in $H_{+}^{0}$, and such that $\left\{z f_{n_{k}}\left(z, \tau_{n_{k}}\right)\right\}$ converges uniformly to $z F(z)$ in every $S_{\delta, \eta}, \delta, \eta>0$. Also, $\left\{f_{n_{k}}\left(z, \tau_{n_{k}}\right)\right\}$ converges uniformly to $F(z)$ on compact subsets of $H_{+}^{0}$. Since $f_{n}\left(z_{0}, \tau_{n}\right)=S_{n}\left(z_{0}, \tau_{n}\right)=\omega_{n}$, we have $F\left(z_{0}\right)=\lim _{n \rightarrow \infty} \omega_{n}=\omega_{0}$.

Let $\varepsilon>0, \delta>0, \eta>0$ be given. We choose $N$ so large that

$$
\left|z f_{n_{k}}\left(z, \tau_{n_{k}}\right)-z F(z)\right|<\varepsilon / 2
$$

for $z \in S_{\delta, \eta}, n_{k} \geq N$. (This is possible by the uniform convergence of $z f_{n_{k}}\left(z, \tau_{n_{k}}\right)$ to $z F(z)$.) We fix one such $n_{k}$ and choose $e \geq \eta$ so large that

$$
\left|z f_{n_{k}}\left(z, \tau_{n_{k}}\right)-\gamma_{-1}^{(\infty)}\right|<\varepsilon / 2
$$

for $z \in S_{\delta, e}$. (This is possible by (4.17) and Remark 4.4.) We therefore have

$$
\left|z F(z)-\gamma_{-1}^{(\infty)}\right|<\varepsilon
$$

for $z \in S_{\delta, e}$. This shows that

$$
F(z)=\gamma_{-1}^{(\infty)} z^{-1}+o\left(z^{-1}\right) .
$$

For each $z \in H_{+}^{0}$ and each $n, f_{n}\left(z, \tau_{n}\right) \in \Delta_{n}(z)$. Consequently $F(z) \in$ $\Delta(z)$, and by Theorem 6.2, $F(z)$ has the expansions (2.19a).

Theorem 6.4. Let a positive collection of power series (2.19) be given. Then the corresponding modified Pick-Nevanlinna problem has solutions. An analytic function which satisfies (2.1b) is a solution iff $F(z) \in \Delta(z)$ for all $z \in H_{+}^{0}$. The problem has a unique solution iff the limit point situation obtains.

Proof. It follows from Theorem 6.3 that the problem always has solutions. In the limit point situation there is exactly one analytic function $F(z)$ satisfying $F(z) \in \Delta(z)$ for all $z \in H_{+}^{0}$, hence by Theorem 6.1 there is exactly one analytic function with the expansions (2.19). For the limit circle situation there is for an arbitrary $z_{0}$ an infinity of analytic functions having the asymptotic expansions (2.19).

Remark 6.5. We observe the following. Let $F_{0}(z)$ be a given Nevanlinna function with the asymptotic expansions (2.19). Then the collection of asymptotic series is positive. It follows that the convergent subsequences of generalized approximants used in the proof of Theorem 6.3 converge to $N$-functions $F(z)$ 
with the same asymptotic expansions as $F_{0}(z)$. (However, no such subsequence need convergence to $F_{0}(z)$.)

\section{THE EXTENDEd HAMBuRger MOMENT PROBLeM}

The extended Hamburger moment problem (associated with the set $\left\{a_{1}, \ldots\right.$, $\left.\left.a_{p}\right\}\right)$ is the following: Let $\left\{c_{j}^{(i)}: j=1,2, \ldots\right\}, i=1, \ldots, p$, be sequences of real numbers and $c_{0}$ a real number. Find distribution functions $\psi(t)$ (i.e., bounded, nondecreasing functions) which have the property

$$
\int_{-\infty}^{\infty} d \psi(t)=c_{0}, \quad \int_{-\infty}^{\infty} \frac{d \psi(t)}{\left(t-a_{i}\right)^{j}}=c_{j}^{(i)}, \quad i=1, \ldots, p, j=1,2, \ldots
$$

(For convenience we do not require here that $\psi(t)$ has infinitely many points of increase.) This problem was treated in $[17,18]$ within the framework of a theory of positive linear functionals and orthogonal rational functions. In this paper we show (without making use of the material in $[17,18]$ ) how the moment problem fits into the framework of the theory of asymptotic expansions of Nevanlinna functions.

We associate with the given sequences the series

$$
\begin{gathered}
\sum_{j=0}^{\infty} c_{j+1}^{(i)}\left(z-a_{i}\right)^{j}, \quad i=1, \ldots, p, \\
-c_{0} z^{-1} .
\end{gathered}
$$

That is; we set

$$
\gamma_{j}^{(i)}=c_{j+1}^{(i)} \quad \text { for } i=1, \ldots, p, j=1,2, \ldots, \quad \gamma_{-1}^{(\infty)}=-c_{0},
$$

and consider the series (2.19) with these coefficients. Asymptotic expansions are as usual in the angular regions $R_{i, \delta}, i=1, \ldots, p$, and $R_{\delta}, \delta>0$.

For each distribution function $\psi(t)$ we define its Stieltjes transform

$$
\hat{\psi}(z)=\int_{-\infty}^{\infty} \frac{d \psi(t)}{t-z} .
$$

We note that $\hat{\psi}(z)$ is a Nevanlinna function.

Theorem 7.1. The following result holds for given $\left\{c_{j}^{(i)}\right\}, c_{0}$ : If the distribution function $\psi(t)$ is a solution of the moment problem (7.1), then the Stieltjes transform $\hat{\psi}(z)$ has the asymptotic expansions

$$
\begin{aligned}
& \hat{\psi}(z) \approx \sum_{j=0}^{\infty} c_{j+1}^{(i)}\left(z-a_{i}\right)^{j} \text { for } i=1, \ldots, p, \\
& \hat{\psi}(z)=-c_{0} z^{1}+o\left(z^{-1}\right) .
\end{aligned}
$$

Proof. Set $z=x+i y$. We then have

$$
z \hat{\psi}(z)=\int_{-\infty}^{\infty} \frac{\left[x(t-x)-y^{2}\right]+i t y}{(t-x)^{2}+y^{2}} d \psi(t) .
$$


We note that $\left(x(t-x)-y^{2}\right) /\left((t-x)^{2}+y^{2}\right)$ is bounded for $z \in R_{\delta}$, and tends to -1 as $z$ tends to $\infty$ in $R_{\delta}$. Consequently,

$$
z \hat{\psi}(z) \rightarrow-\int_{-\infty}^{\infty} d \psi(t)=-c_{0} .
$$

This means that

$$
\hat{\psi}(z)-\left(-c_{0} / z\right)=o\left(z^{-1}\right)
$$

which is $(7.5 b)$.

Next fix an $i \in\{i, \ldots, p\}$. By expanding $1 /(t-z)$ in a finite geometric series in terms of powers of $\left(z-a_{i}\right) /\left(t-a_{i}\right)$ we get

$$
\frac{1}{t-z}=\sum_{j=0}^{n} \frac{\left(z-a_{i}\right)^{j}}{\left(t-a_{i}\right)^{j+1}}+\frac{\left(z-a_{i}\right)^{n+1}}{\left(t-a_{i}\right)^{n+1}(t-z)}
$$

and hence

$$
\hat{\psi}(z)=\sum_{j=0}^{n}\left(z-a_{i}\right)^{j} \int_{-\infty}^{\infty} \frac{d \psi(t)}{\left(t-a_{i}\right)^{j+1}}+\left(z-a_{i}\right)^{n+1} \int_{-\infty}^{\infty} \frac{d \psi(t)}{\left(t-a_{i}\right)^{n+1}(t-z)} .
$$

Thus we have

$$
\hat{\psi}(z)=\sum_{j=0}^{n} c_{j+1}^{(i)}\left(z-a_{i}\right)^{j}+h_{n}^{(i)}(z) \cdot\left(z-a_{i}\right)^{n+1}
$$

where

$$
h_{n}^{(i)}(z)=\int_{-\infty}^{\infty} \frac{d \psi(t)}{\left(t-a_{i}\right)^{n+1}(t-z)} .
$$

It can easily be seen that $|t-z| \geq\left(t-a_{i}\right) \sin \delta$, hence

$$
\left|h_{n}^{(i)}(z)\right| \leq \frac{1}{\sin \delta} \int_{-\infty}^{\infty} \frac{d \psi(t)}{\left|t-a_{i}\right|^{n+2}}
$$

when $z \in R_{i, \delta}$. Note that

$$
\int_{-\infty}^{\infty} d \psi(t) /\left|t-a_{i}\right|^{n+2}<\infty
$$

since $\int_{-\infty}^{\infty} d \psi(t) /\left(t-a_{i}\right)^{n+2}$ is assumed to exist. Thus there exists a constant $s_{i, n}$ such that

$$
\left|\hat{\psi}(z)-\sum_{j=0}^{n} c_{j+1}^{(i)}\left(z-a_{i}\right)^{j}\right| \leq s_{i, n}\left|z-a_{i}\right|^{n+1}
$$

when $z \in R_{i, \delta}$.

This shows that $\hat{\psi}(z)$ has the asymptotic expansions (7.5b). 
Theorem 7.2. The following result holds for given $\left\{c_{j}^{(i)}\right\}, c_{0}$ : If the distribution function $\psi(t)$ is such that its Stieltjes transform $\hat{\psi}(z)$ has the asymptotic expansions (7.5a)-(7.5b), then $\psi(t)$ is a solution of the moment problem (7.1).

Proof. Again set $z=x+i y$. We then have

$$
\operatorname{Re}(i y \hat{\psi}(i y))=\int_{-\infty}^{\infty} \frac{-y^{2}}{t^{2}+y^{2}} d \psi(t) .
$$

It follows from (7.5b) that $i y \hat{\psi}(i y) \rightarrow-c_{0}$, and since $c_{0}$ is real, this implies

$$
\operatorname{Re}(i y \hat{\psi}(i y)) \rightarrow-c_{0} \text { as } y \rightarrow \infty .
$$

The family $y^{2} /\left(t^{2}+y^{2}\right)$ tends to 1 monotonically as $y$ tends to $\infty$, and therefore

$$
\int_{-\infty}^{\infty} \frac{y^{2}}{t^{2}+y^{2}} d \psi(t) \rightarrow \int_{-\infty}^{\infty} d \psi(t) \quad \text { as } y \rightarrow \infty
$$

From (7.12), (7.13), and (7.14) we conclude that

$$
\int_{-\infty}^{\infty} d \psi(t)=c_{0}
$$

Let $k$ be a given index. We have

$$
\operatorname{Re}\left(\hat{\psi}\left(a_{k}+i y\right)\right)=\int_{-\infty}^{\infty} \frac{\left(t-a_{k}\right) d \psi(t)}{\left(t-a_{k}\right)^{2}+y^{2}} .
$$

It follows from (7.5a) that

$$
\hat{\psi}\left(a_{k}+i y\right) \rightarrow c_{1}^{(k)} \text { as } y \rightarrow 0 .
$$

Since $c_{1}^{(k)}$ is real, this implies

$$
\operatorname{Re}\left(\hat{\psi}\left(a_{k}+i y\right)\right) \rightarrow c_{1}^{(k)} \text { as } y \rightarrow 0 .
$$

By considering the cases $t<a_{k}$ and $t>a_{k}$ separately, we see that

$$
\frac{t-a_{k}}{\left(t-a_{k}\right)^{2}+y^{2}}
$$

tends to $1 /\left(t-a_{k}\right)$ monotonically when $y$ tends to zero, and therefore

$$
\int_{-\infty}^{\infty} \frac{\left(t-a_{k}\right) d \psi(t)}{\left(t-a_{k}\right)^{2}+y^{2}} \rightarrow c_{1}^{(k)} \text { as } y \rightarrow 0 .
$$

It follows from (7.16), (7.18), and (7.15) that

$$
\int_{-\infty}^{\infty} \frac{d \psi(t)}{t-a_{k}}=c_{1}^{(k)}
$$

Now assume that for some $n$ it is known that

$$
\int_{-\infty}^{\infty} \frac{d \psi(t)}{\left(t-a_{k}\right)^{j+1}}=c_{j+1}^{(k)}, \quad j=0, \ldots, n .
$$


It follows from (7.5a) that

$$
\frac{1}{\left(z-a_{k}\right)^{n+1}}\left(\hat{\psi}(z)-\sum_{j=0}^{n} c_{j+1}^{(k)}\left(z-a_{k}\right)^{j}\right) \rightarrow c_{n+2}^{(k)}
$$

when $z \rightarrow a_{k}$ in $R_{k, \delta}$. This means that

$$
\frac{1}{\left(z-a_{k}\right)^{n+1}} \int_{-\infty}^{\infty}\left(\frac{1}{t-z}-\sum_{j=0}^{n} \frac{\left(z-a_{k}\right)^{j}}{\left(t-a_{k}\right)^{j+1}}\right) d \psi(t)
$$

tends to $c_{n+2}^{(k)}$ when $z$ tends to $a_{k}$ in $R_{k, \delta}$. By summing the finite geometric series under the integral sign we can write this as

$$
\int_{-\infty}^{\infty} \frac{d \psi(t)}{(t-z)\left(t-a_{k}\right)^{n+1}} \rightarrow c_{n+2}^{(k)} .
$$

Since $c_{n+2}^{(k)}$ is real, the real part of the left-hand side of (7.23) tends to $c_{n+2}^{(k)}$, hence

$$
\int_{-\infty}^{\infty} \frac{d \psi(t)}{\left[\left(t-a_{k}\right)^{2}+y^{2}\right]\left(t-a_{k}\right)^{n}} \rightarrow c_{n+2}^{(k)} \text { as } y \rightarrow 0 .
$$

Again $1 /\left[\left(t-a_{k}\right)^{2}+y^{2}\right]\left(t-a_{k}\right)^{n}$ tends to $1 /\left(t-a_{k}\right)^{n+2}$ monotonically for $t<a_{k}$ and $t>a_{k}$ when $y$ tends to zero, and therefore

$$
\int_{-\infty}^{\infty} \frac{d \psi(t)}{\left[\left(t-a_{k}\right)^{2}+y^{2}\right]\left(t-a_{k}\right)^{n}} \rightarrow \int_{-\infty}^{\infty} \frac{d \psi(t)}{\left(t-a_{k}\right)^{n+2}} .
$$

Thus by (7.24) and (7.25) we have

$$
\int_{-\infty}^{\infty} \frac{d \psi(t)}{\left(t-a_{k}\right)^{n+2}}=c_{n+2}^{(k)} .
$$

It follows by induction that all the moments of $\psi(t)$ exist, and that $\psi(t)$ is a solution of the moment problem.

Theorem 7.3. Let a positive collection of power series (2.19) be given. Then the Stieltjes transforms of the solutions of the moment problem (7.1) are exactly those analytic functions $F(z)$ in $H_{+}^{0}$ for which (7.5b) is satisfied and for which $F(z) \in \Delta(z)$ for all $z \in H_{+}^{0}$. Equivalently the Stieltjes transforms are those analytic functions $F(z)$ in $H_{+}^{0}$ which have the asymptotic expansions (7.5). Such functions exist. The moment problem has a unique solution iff the limit point situation obtains.

Proof. Let $F(z)$ be a Nevanlinna function with the series (7.2) as asymptotic expansions. From the expansion $F(z)=-c_{0} z^{-1}+o\left(z^{-1}\right)$ it follows in particular that for $y$ real,

$$
\sup _{y \geq 1}|i y F(i y)|<\infty .
$$


Then there exists a distribution function $\psi(t)$ such that $F(z)=\hat{\psi}(z)$ (see, e.g., [1, pp. 92-94]). Thus all Nevanlinna functions with the series (7.2) as asymptotic expansions are Stieltjes transforms of distribution functions. The theorem now follows by combining Theorems 6.1-6.4 and Theorems 7.1-7.2.

\section{REFERENCES}

1. N. I. Akhiezer, The classical moment problem and some related questions in analysis, Hafner, New York, 1965.

2. C. Carathéodory, Über den Variabilitätsbereich der Koeffizieten von Pontenzreihen die gegebenen Werte nicht annehmen, Math. Ann. 64 (1907), 95-115.

3. _ـ Über den Variabilitatsbereich der Fouriersche Funktionen, Rend. Circ. Mat. Palermo 32 (1911), 193-217.

4. A. Dinghas, Vorlesungen über Funktionentheorie, Springer-Verlag, Berlin, 1961.

5. I. Gohberg (Ed.), I. Schur methods in operator theory and signal processing, Operator Theory: Advances and Applications, No. 18, Birkhauser, Basel-Boston-Stuttgart, 1986.

6. H. Hamburger, Über eine Erweiterung des Stieltjesschen Momentproblems, Math. Ann. 81 (1920), 235-319; 82 (1921), 120-164; 82 (1921), 168-187.

7. E. Hendriksen and $\mathrm{O}$. Njảstad, A Favard theorem for rational functions, J. Math. Anal. Appl. 142 (1989), 508-520.

8. 261-269.

9. P. Henrici, Applied and computatonal complex analysis, Vol. II, Wiley, New York, 1977.

10. W. B. Jones, O. Njästad, and W. J. Thron, Continued fractions and strong Hamburger moment problems, Proc. London Math. Soc. (3) 47 (1983), 363-384.

11. __ Orthogonal Laurent polynomials and the strong Hamburger moment problem, J. Math. Anal. Appl. 98 (1984), 528-584.

12. _ Moment theory, orthogonal polynomials, quadrature, and continued fractions associated with the unit circle, Bull. London Math. Soc. 21 (1989), 113-152.

13. W. B. Jones and W. J. Thron, Continued fractions: Analytic theory and applications, Encyclopedia of Mathematics and its Applications, No. 11, Addison-Wesley, Reading, Mass., 1980. (Now distributed by Cambridge Univ. Press.)

14. M. G. Krein and P. G. Rekhtman, On the Nevanlinna-Pick problem, Trudy Odess. Gos. Univ. 2 (1938), 63-68. (Ukranian)

15. R. Nevanlinna, Über beschränkte Funktionen, die in gegebenen Punkten vorgescriebene Werte annehmen, Ann. Acad. Sci. Fenn. Ser. A I Math. 13 (1919), No. 1.

16. _ـ Über beschränkte analytische Funktionen, Ann. Acad. Sci. Fenn. Ser. A I Math. 32 (1929), No. 7.

17. O. Njåstad, An extended Hamburger moment problem, Proc. Edinburgh Math. Soc. (2) 28 (1985), 167-183.

18. __ Unique solvability of an extended Hamburger moment problem, J. Math. Anal. Appl. 124 (1987), 502-519.

19. __ Multipoint Padé approximation and orthogonal rational functions, Nonlinear Numerical Methods and Rational Approximation (A Cuyt, ed.), Reidel, Dordrecht, 1988, pp. 259-270.

20. __ Laurent continued fractions corresponding to pairs of power series, J. Approx. Theory 55 (1988), 119-139.

21. __ Asymptotic expansions and contractive Laurent fractions, Proc. London Math. Soc. (3) 56 (1988), 78-100. 
22. __ Solution of the strong Hamburger moment problem by Laurent continued fractions, Appl. Numer. Math. 4 (1988), 351-360.

23. _ Contractive Laurent fractions and nested discs, J. Approx. Theory 56 (1989), 134-151.

24. __ Multipoint Padé approximants and related continued fractions, Analytic Theory of Continued Fractions III (L. Jacobsen, ed.), Lecture Notes in Math., vol. 1406, SpringerVerlag, 1989, pp. 76-87.

25. O. Njåstad and W. J. Thron, Unique solvability of the strong Hamburger moment problem, J. Austral. Math. Soc. Ser. A 40 (1986), 5-19.

26. O. Perron, Die Lehre von den Kettenbrüchen, Bd. 2, 3, Auflage, Teubner, Stuttgart, 1957.

27. G. Pick, Über Beschrankungen analytischer Funktionen, welche durch vorgegebenen Funktionswerte bewirkt sind, Math. Ann. 77 (1916), 7-23.

28. __ Über beschränkte Funktionen mit vorgegebenen Wertzuordnungen, Ann. Acad. Sci. Fenn. Ser. A I Math. 15 (1920), No. 3.

29. D. Sarason, Generalized interpolation in $H^{\infty}$, Trans. Amer. Math. Soc. 127 (1967), 179203.

30. _ Operator theoretic aspects of the Nevanlinna-Pick interpolation problems, Operators and Function Theory (S. C. Power, ed.), Reidel, Dordrecht, 1985.

31. I. Schur, Über Potenzreihen die im Innern des Einheitskreises beschränkt sind, J. Reine Angew. Math. 147 (1917), 205-232; 148 (1918-19), 122-145.

32. J. A. Shohat and J. D. Tamarkin, The problem of moments, Math. Surveys, No. 1, Amer. Math. Soc., Providence, R.I., 1943.

33. O. Toeplitz, Über die Fouriersche Entwiklung positiver Funktionen, Rend. Circ. Mat. Palermo 32 (1911), 191-192.

34. H. Wallin, Rational interpolation to meromorphic functions, Padé Approximation and its Applications (M. G. de Bruin and H. van Rossum, eds.), Lecture Notes in Math., vol. 888, Springer-Verlag, 1981, pp. 371-382.

Department of Mathematics, University of TRONDheim-NTH, N-7034 Trondheim, NoRWAY 